CERN-TH/97-199

MPI-PhT/97-68

November 1997

\title{
Perturbative Universality in Soft Particle Production
}

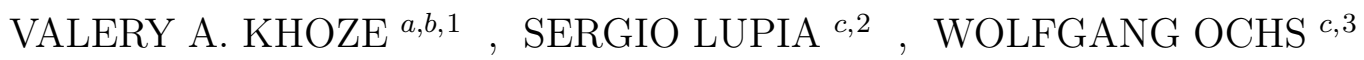 \\ a INFN - Laboratori Nazionali di Frascati, P.O. Box 13, \\ I-00044 Frascati, Italy \\ b TH Division CERN \\ CH-1211, Geneva 23 \\ c Max-Planck-Institut für Physik \\ (Werner-Heisenberg-Institut) \\ Föhringer Ring 6, D-80805 Munich, Germany
}

\begin{abstract}
The spectrum of partons in a QCD jet becomes independent of the primary energy in the low momentum limit. This follows within the perturbative QCD from the colour coherence in soft gluon branching. Remarkably, the hadrons follow such behaviour closely, suggesting the parton hadron duality picture to be appropriate also for the low momentum particles. More generally, this scaling property holds for particles of low transverse and arbitrary longitudinal momentum, which explains an old experimental observation ("fan invariance"). Further tests of the perturbatively based picture for soft particle production are proposed for three-jet events in $e^{+} e^{-}$annihilation and di-jet production events in $\gamma p, \gamma \gamma$ and $p \bar{p}$ collisions. They are based upon the difference in the intensity of the soft radiation from primary $q \bar{q}$ and gg antennae.
\end{abstract}

\footnotetext{
${ }^{1}$ e-mail: khoze@vxcern.cern.ch

2e-mail: lupia@mppmu.mpg.de

3 e-mail: wwo@mppmu.mpg.de
} 


\section{Introduction}

The study of multiparticle production in hard processes provides one with an important information about both the QCD partonic branching processes and the transition from the coloured partons to the colourless hadrons. The vast amount of experimental data collected in various hard collisions processes convincingly confirms that the inclusive characteristics of QCD jet systems can be successfully described within the analytical perturbative approach to multiparticle production, see e.g. [1, 2, 3]. This approach is based on the so-called Modified Leading Logarithmic Approximation (MLLA) [4.5] and on the concept of Local Parton Hadron Duality (LPHD) [6].

In particular, the experiment clearly demonstrates that the bright colour coherence effects survive the hadronization stage and are distinctively visible in the data. This has been known for quite a long time for $e^{+} e^{-}$annihilation processes (see e.g. [3]). Recently the very impressive data from HERA [7,8,9] and from the TEVATRON 10,11] have become available.

The LPHD allows one to relate the (sufficiently) inclusive hadronic observables to the corresponding quantities computed for the cascading partonic system. One of the well known (but still striking) predictions of the perturbative scenario is the depletion of the soft particle production and the resulting approximately Gaussian shape of the inclusive distribution in the variable $\xi=\ln (P / E)$ for particles with energy $E$ in a jet of energy $P$ (the so-called "hump-backed plateau") 6, 12,13.

This behaviour is observed in all inclusive energy distributions; the charged particle spectrum for not too small momenta prove to be in surprisingly good agreement with the MLLA-LPHD predictions reflecting the QCD cascading picture of multiple hadroproduction. Moreover, the data collected in various hard scattering processes $\left(e^{+} e^{-}\right.$, DIS, $\left.\bar{p} p\right)$ clearly demonstrate a remarkable universality of particle spectra assuming the proper (MLLA-based) choice of the cascading evolution variables, equivalent to the $e^{+} e^{-} \mathrm{cms}$ energy $\sqrt{s}$.

The most challenging is the soft momentum end of the particle spectra $(\mathrm{p} \lesssim 1 \mathrm{GeV})$ where the non-perturbative dynamics could invalidate the perturbatively based expectations. An attempt to stretch the perturbative predictions to the limit of their applicability (or better to say, beyond it) has been performed in previous papers of the present authors (see e.g. 14, 15, 16, 17]). In these papers it was shown that (after the proper modifications) the perturbatively based formulae allow a sufficiently smooth transition into the soft momentum domain. The non-perturbative hadronization effects are encoded in the transverse momentum cutoff $Q_{0}$ which can be motivated by the space-time picture of hadroproduction in QCD jets, see e.g. [2]. Explicit pre-

dictions for distributions of hadrons in the soft region need some additional 
assumptions on mass effects.

Scaling phenomena are expected to be of more general nature. Let us recall that the gluons of long wave length are emitted by the total colour current which is independent of the internal structure of the jet and is conserved when the partons split. Applying the LPHD hypothesis one then expects that the hadron spectrum at very low momenta $p_{h}$ should be nearly independent of the jet energy [6, 14, 18].

As discussed in [15], the low momentum data support the basic ideas of QCD coherence and LPHD. Quantitatively, the analysis was performed in terms of the invariant particle density $E \frac{d n}{d^{3} p}$ for $e^{+} e^{-}$annihilation into hadrons at low momenta in quite a wide cms energy region (from ADONE to LEP-2). The spectra were found to be in a good agreement with the scaling behaviour and with analytical perturbative expectations which became sensitive to the running of the coupling $\alpha_{s}$ at small scales [14,15]. The new H1 data 8] follow these predictions as well, thus confirming the universality of soft particle production.

In this paper we study further the phenomenology of the soft particle production for both the charged and identified hadrons. The analytical calculations are compared with the quantity $\frac{d n}{d^{3} p}$ which has some practical advantages over the invariant density considered before. We derive a scaling prediction for particles at low transverse but arbitrary longitudinal momenta, which generalizes our previous results and which for very small $p_{\perp}$ explains the so-called "fan-invariance" experimentally observed long ago [19].

An important test of this line of approach towards soft particle production is the sensitivity to the colour charge of the primary emitters: the density of low momentum particles roughly doubles when going from a primary $q \bar{q}$ to a $g g$ antenna. It is interesting to note that this prediction applies to the soft particles already at present energies, whereas for the total multiplicity this doubling needs much higher energies. The sensitivity of the low momentum particle density to the primary colour charges can be studied in experimentally accessible processes, such as in $e^{+} e^{-} \rightarrow 3$ jets [15]. Here this process is discussed further and we derive predictions for the soft particle production in quark and gluon exchange reactions which are observed in photoproduction or DIS at HERA or in $\gamma \gamma$ collisions. 


\section{Perturbative predictions for particle pro- duction in the soft limit}

In refs. [15, 16] an approximate solution of the MLLA evolution equation for the inclusive energy distribution $D_{A}^{g}$ of soft gluons, originating from a primary parton $A$, has been derived. This solution can be written as

$$
D_{A}^{g}(\xi, Y)=\left.\frac{C_{A}}{N_{C}} D_{g}^{g}(\xi, Y)\right|_{D L} \exp (-G) \quad, \quad G=\frac{a}{4 N_{C}} \int_{\xi}^{Y} \gamma_{0}^{2}(y) d y
$$

with

$$
\left.D_{g}^{g}(\xi, Y)\right|_{D L}=\beta^{2} \ln \frac{Y-\xi+\lambda}{\lambda}+\beta^{4} \int_{0}^{Y-\xi} d \tau \ln \frac{\tau+\lambda}{\lambda} \ln \frac{\tau+\xi+\lambda}{\tau+\lambda}+\cdots
$$

Here we have used the logarithmic variables $\xi=\ln (1 / x)=\ln (Q / E), Y=$ $\ln \left(Q / Q_{0}\right)$ and $\lambda=\ln \left(Q_{0} / \Lambda\right)$ with $E$ the particle energy and $Q$ the jet virtuality $(Q=P \Theta$ for a jet of primary momentum $P$ and half opening angle $\Theta) ; C_{A}$ is the respective colour factor, i.e., $C_{g}=N_{C}$ and $C_{q}=C_{F} ; \gamma_{0}$ denotes the anomalous dimension of multiplicity and is related to the $Q C D$ running coupling by $\gamma_{0}^{2}=4 N_{C} \alpha_{s} / 2 \pi$ or $\gamma_{0}^{2}=\beta^{2} / \ln \left(p_{\perp} / \Lambda\right)$ with $\beta^{2}=4 N_{C} / b$, $b \equiv\left(11 N_{C}-2 n_{f}\right) / 3 ; \Lambda$ is the QCD-scale and $N_{C}$ and $n_{f}$ are the numbers of colours and of flavours respectively, $a=\frac{11}{3} N_{C}+\frac{2 n_{f}}{3 N_{C}^{2}}$. The shower evolution is terminated by the transverse momentum cut-off $p_{\perp} \geq Q_{0}$.

The first term in (2) corresponds to the emission of a single gluon and yields the leading contribution for $E \rightarrow Q_{0}$. It is proportional to the colour charge of the primary parton. Furthermore, this term does not depend on the cms energy, contrary to the higher order terms which provide the rise of the spectrum for large $E$ with increasing $\sqrt{s}$. The spectrum vanishes at $E \rightarrow Q_{0}$ as

$$
D_{A}^{g}(\xi, Y) \approx \frac{C_{A}}{N_{C}} \beta^{2} \frac{\left(E-Q_{0}\right)}{\lambda Q_{0}}
$$

In order to relate parton and hadron distributions at low momentum $p \sim Q_{0}$, one has to make some additional assumptions on how to include

mass effects. In 14, 15 it was required that the invariant density $E_{h} \frac{d n}{d^{3} p_{h}}$ for hadrons approaches a constant limit when $p_{h} \rightarrow 0$ as observed experimentally. Here it is worth to recall that at low $p_{h}$

$$
E_{h} \frac{d n}{d^{3} p_{h}} \sim \frac{\overline{W_{1}}\left(s, E_{h} \sqrt{s}\right)}{s},
$$


where $\overline{W_{i}}\left(s, E_{h} \sqrt{s}\right)$ are the standard $e^{+} e^{-}$analogues of the DIS structure functions $W_{i}\left(q^{2}, \nu\right)$, see e.g. 20]. As well known, $\bar{W}_{i}\left(s, E_{h} \sqrt{s}\right)$ are related to the matrix elements of the current commutators and should be regular when $p_{h} \rightarrow 0$.

For spectra which vanish as in (3) a plausible relation between partons and hadrons can be achieved by relating their distributions as [12, 14]

$$
E_{h} \frac{d n_{A}\left(\xi_{E}\right)}{d p_{h}}=K_{h} E_{p} \frac{d n_{A}\left(\xi_{E}\right)}{d p_{p}} \equiv K_{h} D_{A}^{g}\left(\xi_{E}, Y\right)
$$

at the same energies $E_{p}=E_{h}=\sqrt{p_{h}^{2}+Q_{0}^{2}} \equiv E \geq Q_{0}$ where $\xi_{E} \equiv \xi=$ $\ln (Q / E)$ and $K_{h}$ is a normalization parameter. If hadrons from both hemispheres are added, $K_{h}$ should be replaced by $2 K_{h}$. For large energies $E_{h} \gg$ $Q_{0}$ (5) coincides with the usual LPHD relation $d n / d \xi=K_{h} D(\xi, Y)$.

In [15] we have considered the invariant density $E d n / d^{3} p \equiv d n / d y d^{2} p_{T}$ in the limit of vanishing rapidity $y$ and transverse momentum $p_{\perp}$ or, equivalently, for vanishing momentum $|\vec{p}| \equiv p$, i.e.,

$$
I_{0}=\lim _{y \rightarrow 0, p_{T} \rightarrow 0} E \frac{d n}{d^{3} p}=\frac{1}{2} \lim _{p \rightarrow 0} E \frac{d n}{d^{3} p}
$$

where the factor $1 / 2$ takes into account that both $e^{+} e^{-}$hemispheres are added in the limit $p \rightarrow 0$.

Then, since $E_{h}-Q_{0} \approx p_{h}^{2} /\left(2 Q_{0}\right)$ for small $p_{h}$, the invariant hadronic density

$$
E_{h} \frac{d n}{d^{3} p_{h}}=K_{h} D_{A}^{g}(\xi, Y) /\left(4 \pi p_{h}^{2}\right)
$$

approaches the finite, energy independent limit as in (6)

$$
I_{0}=K_{h} \frac{C_{A} \beta^{2}}{8 \pi N_{C} \lambda Q_{0}^{2}} .
$$

In the fixed $\alpha_{s}$ limit $\beta^{2} / \lambda$ is replaced by $\gamma_{0}$ and $I_{0} \sim 1 / Q_{0}^{2}$. With prescription (5) one obtains a good description of the moments of the energy spectrum $D(\xi, Y)$ by the MLLA formulae with $Q_{0}=270 \mathrm{MeV}$ in a wide energy range [14, as well as of the particle spectrum in the soft region $\left(E_{h}<1 \mathrm{GeV}\right)$ [15].

Certainly at the moment there is no unique recipe allowing one to perform a smooth transition between the perturbative and non-perturbative regimes. Also there are certain approximations involved in the analytical results for low energy particles. In refs. [21,22] an alternative prescription has been discussed:

$$
\frac{d n}{d \ln p}=\left.\left(\frac{p}{E}\right)^{3} D_{A}^{g}\left(\xi_{E}, Y\right)\right|_{\mathrm{LS}}
$$


where $\left.D_{A}^{g}\left(\xi_{E}, Y\right)\right|_{\text {LS }}$ is the so-called Limiting Spectrum [4, [6] which solves the MLLA evolution equations for $\lambda=0$ in the full kinematic region, except very close to the boundary $\xi_{E}=\mathrm{Y}$. At this phase space boundary the Limiting Spectrum approaches the finite limit $D_{g}^{g}(Y, Y)=\frac{4 N_{C}}{a}$, which equals numerically 1.069 (1.055) for $n_{f}=3(5)$.

Basing on the prescription (9) one arrives at the alternative formula for the invariant hadronic density which in the case of $e^{+} e^{-}$annihilation reads

$$
E_{h} \frac{d n}{d^{3} p_{h}}=\left.2 K_{h}\left(\frac{1}{4 \pi E_{h}^{2}}\right) D_{q}^{g}\left(\xi_{E}, Y\right)\right|_{\mathrm{LS}},
$$

with $D_{q}^{g}=\frac{C_{F}}{N_{C}} D_{g}^{g}$. As it is easy to see this prescription also guarantees that the hadronic density reaches a constant limit at $p_{h} \rightarrow 0$. We shall compare here the expectation based on Eq. (10) with the experimental data for charged pions $\left(K_{h}=K_{\pi}\right)$. The MLLA Limiting Spectrum taken at $\Lambda=Q_{0}=150 \mathrm{MeV}$ has been found to provide a good description of pion spectra at relativistic energies, see e.g. [6,22]. Recall that the low momentum region in charged particle spectra is dominated by the pions.

\section{Phenomenology of soft particle production}

The energy dependence of the soft particle production rate was investigated previously for $e^{+} e^{-}$annihilation in terms of the invariant particle density $E d n / d^{3} p$ in the wide energy interval (from ADONE to LEP-2) [15]. Recently, new data on soft particle production became available from DIS at HERA [8]. In the following we compare various results for the quantity $d n / d^{3} p$ as a function of the momentum $p$ of the registered particle. When presented in terms of this observable the data points are independent of the choice of the theoretical parameter $Q_{0}$; in this case, however, we are dealing with a quantity which is not directly related to the Lorentz-invariant phase space. Using this quantity may facilitate the comparison between different experimental results.

\subsection{Energy dependence of soft particle production}

Fig. 1 shows the momentum dependence of $d n / d^{3} p$ for various $\mathrm{cms}$ energies $\sqrt{s}$ in $e^{+} e^{-}$annihilation in comparison with the theoretical predictions. In Fig. 1a the distributions of charged particles are presented in comparison with the approximate MLLA result (11) with prescription (5) for the relation

\footnotetext{
${ }^{4}$ We are grateful to Yu. L. Dokshitzer for recovering this result.
} 
between partons and hadrons. The theoretical prediction describes well the approach to the scaling limit for low momenta and also the shape and energy dependence of the spectra with the choice $Q_{0}=270 \mathrm{MeV}$, as already discussed in [15]. The detailed behaviour at the very small momenta $p \lesssim Q_{0}$ depends on the approximation at the parton level involving large production angles $\left(\vartheta>Q_{0} / p\right)$ and also on the assumed relation between partons and hadrons in this region.

This point is illustrated in Fig. 1b, where we show the theoretical predictions with the alternative choice of the low momentum behaviour discussed in Sect. 2, based on the approximate analytical MLLA solution for $Q_{0}=\Lambda$ (Limiting Spectrum) and relation (9); this form describes the pion spectra for $\sqrt{s} \gtrsim 10 \mathrm{GeV}$ with the mass parameter $Q_{0}=150 \mathrm{MeV}$ which is in agreement with the earlier fits to the pion spectra 6, 22]. With this prescription the particle production rate in the soft limit $p \rightarrow 0$ is lower than in Fig. 1a, but it also approaches an energy independent value in this limit and that is the essential prediction of the QCD approach based on coherent soft gluon emission.

Looking at the data in Fig. 1 more closely, there seems to be a certain increase of the soft particle rate at LEP energies. This is illustrated in Fig. $2 \mathrm{a}$ and b where the data below and above $\sqrt{s}=91 \mathrm{GeV}$ respectively in $e^{+} e^{-}$annihilation show separately a very good approach to an energy independent limit at small $p$, whereas there is a difference in absolute rates of $20-30 \%$ in the two energy regions. This is also visible in Fig. 3a where we show the cms energy dependence of three values of the momentum $p$. We also include here the recent data from HERA [8] which closely match the $e^{+} e^{-}$results. At the lowest momentum $p=0.2 \mathrm{GeV}$ we see an almost energy independent production rate, but a small jump at the LEP-1 energy is visible. A rise at the higher energies could partially be caused by the weak decay products of heavy quark particles (perhaps also particles with strange quarks) which would add incoherently to the spectrum of particles produced directly from the soft gluons. This could happen in particular at the $Z^{0}$ because of the larger branching ratio into $b$-quarks. However, the experiment 23] clearly shows that at low momenta $(p \leq 0.5 \mathrm{GeV})$ the effect induced by the $b \bar{b}$ events is below $5 \%$. It would be interesting to clarify quantitatively in more details which part of the observed rise could be explained by the overall effect of the weak decays. A certain small increase of the rate is actually also expected from the theory at the nonzero momenta (see the curves in Fig. 3a). For larger momenta the rise of the production rate with energy is more pronounced and this trend is reproduced by the theoretical calculation for the full range of energy where the data are available $(\sqrt{s} \gtrsim 3 \mathrm{GeV})$.

An approximate energy independence of the rate for the low momentum 
kaons and protons is well tested for $\sqrt{s} \gtrsim 10 \mathrm{GeV}$ where data at some common low momentum value are available. A comparison in Figs. 3a and $3 \mathrm{~b}$ suggests that the spectra become practically energy independent when the particle momenta are of the order of their mass or smaller.

It should be noted that the energy independence of the soft particle rate is by no means trivial. We recall, in particular, that the particle density $d n / d y$ at central rapidity $y \approx 0$ rises with $\mathrm{cms}$ energy. This is illustrated in Fig. 4 for TASSO and OPAL data where rather precise results are available at $p=0.25 \mathrm{GeV}$. Whereas in this energy range $(14$ to $91 \mathrm{GeV})$ the plateau height rises by about $70 \%$ the corresponding rise for the soft particles at $p=0.25 \mathrm{GeV}$ is only about $10 \%$. This is consistent with the QCD picture of particle production: the central plateau rises asymptotically with the same exponent $\sim \sqrt{\ln s}$ as the full particle multiplicity and this rise comes mainly from the high transverse momentum particles. The soft particle production is suppressed because of the coherence in the emission from all colour sources.

\subsection{Universal soft limit for different particle species?}

In Fig. 5 we consider for different identified particles with mass $m$ the rescaled spectra $m^{3} d n / d^{3} p$ which are expected to approach energy independent dimensionless numbers in the soft limit $p \rightarrow 0$. First we see again, that in the pion spectra the violation of energy independence occurs at lower momenta than in the spectra of the heavier particles. Furthermore, these rescaled spectra of pions and kaons, separated by an order of magnitude around $p \sim 0.8$ $\mathrm{GeV}$ seem to converge towards a common value in the soft limit. A similar convergence is also suggested for the protons although the lack of precise data at small momenta exclude a firm conclusion. We find an approximate universality of the quantity

$$
I_{0}^{m}=\left.m^{3} \frac{d n}{d^{3} p}\right|_{p \rightarrow 0} \approx 0.1 \div 0.2
$$

for the particle species considered here. This corresponds to our previous observation 15 that the invariant density behaves like $I_{0}=E d n /\left.d^{3} p\right|_{p \rightarrow 0} \sim$ $m^{-2}$ in the soft limit. In our theoretical picture this quantity is given by $I_{0} \sim 1 / Q_{0}^{2}$ (and not $1 / \Lambda^{2}$, for example). To the extent that $Q_{0}$ is related to the particle mass such a universality could be anticipated. It would be interesting to have more experimental information on low momentum particles to test better the validity of such a universal behaviour; new HERA data with identified particles at such small momenta could be helpful as well. 


\section{Scaling properties of particle production at small $p_{\perp}$}

\subsection{Colour coherence and "fan invariance"}

As discussed before the gluon spectrum in the soft limit becomes independent of energy of the primary parton above about $1 \mathrm{GeV}$. This energy independence can be observed, for example, in the $\mathrm{cms}$ of $e^{+} e^{-}$annihilation or in the Breit frame of DIS. This result can be easily generalized. We make a Lorentz transformation along the initial parton direction, then in the new frame the initial partons have different energies but the soft particle production rate should remain unaltered as long as the primary energies are still sufficiently large (and the colour of the primary parton has not changed). Consequently, the quantity

$$
I_{0}(y)=\lim _{p_{\perp} \rightarrow 0} E \frac{d n}{d^{3} p}=\lim _{p_{\perp} \rightarrow 0} E \frac{d n}{d y d^{2} p_{\perp}}
$$

should be independent of rapidity $y$ for such transformations 15 and this behaviour should still hold approximately for $p_{\perp}$ not much larger than the hadron masses. Therefore, if the coherence argument is applied to this class of frames one obtains the prediction of scaling for the distribution

$$
\frac{d^{2} n}{d y d p_{\perp}}=f\left(y, p_{\perp}\right) .
$$

Equivalently, for $y \approx \eta=-\ln \operatorname{tg}(\vartheta / 2)$ with production angle $\vartheta$ one expects

$$
\frac{d^{2} n}{d p_{\|} d \vartheta}=f\left(p_{\|}, \vartheta\right)
$$

An approximate scaling behaviour of this type - but for the distributions normalized to unity - was found by the TASSO collaboration [19] in the energy range from 14 to $34 \mathrm{GeV}$ long ago and was called "fan invariance".f] In the following we will show explicitly how this scaling behaviour arises in the perturbative QCD calculations; furthermore we predict how the abovementioned scaling behaviour is violated. This will lead to the suggestion of other variables in which scaling is restored in a wider kinematical region.

\footnotetext{
${ }^{5}$ We thank P. Mättig for pointing out to us this scaling law which triggered the study in this section.
} 


\subsection{Scaling properties of the analytical results}

The double differential distribution $d n / d \xi d \vartheta$ in $\xi=\ln (1 / x)$ and angle $\vartheta$ is derived from the $\xi$-distribution of particles $D(\xi, Y)$ in a jet of half opening angle $\Theta$ by (see also [12])

$$
\frac{d^{2} n}{d \xi d \ln \vartheta}=\left.\frac{d}{d \ln \vartheta} D_{A}^{g}(\xi, Y)\right|_{\Theta=\vartheta} .
$$

with $Y=\ln \left(P \Theta / Q_{0}\right)$ for small angles. For larger angles an appropriate variable is $\tilde{Y}=\ln \left(2 P \sin (\Theta / 2) / Q_{0}\right)$ instead of $Y$ [35]. For the application at small $p_{\perp}$ we take the approximate expression (1) for $D_{A}^{g}(\xi, Y)$ and obtain (with $p_{\perp}=E \vartheta$ )

$$
\frac{d^{2} n}{d \xi d \ln p_{\perp}}=\left.\frac{d}{d Y} D_{A}^{g}(\xi, Y)\right|_{D L} e^{-G}-\frac{\partial G}{\partial Y} D_{A}^{g}(\xi, Y) .
$$

Within the DLA this distribution (the first factor in the first term) is given by

$$
\begin{gathered}
\frac{d^{2} n}{d \xi d \ln p_{\perp}}=\frac{C_{A} \beta^{2}}{N_{C}}\left[\frac{1}{\ln \frac{p_{\perp}}{\Lambda}}+\beta^{2} \ln \left(\frac{\ln \frac{p_{\perp}}{\Lambda}}{\ln \frac{Q_{0}}{\Lambda}}\right) \ln \left(\frac{\ln \frac{P_{\perp}}{\Lambda}}{\ln \frac{p_{\perp}}{\Lambda}}\right)\right] \\
Y-\xi+\lambda=\ln \frac{p_{\perp}}{\Lambda}, \quad Y+\lambda=\ln \frac{P_{\perp}}{\Lambda}=\ln \frac{p_{\perp} / x}{\Lambda} .
\end{gathered}
$$

For small $p_{\perp}$ the first term in the brackets from the single gluon bremsstrahlung dominates and is energy independent. This is the term which corresponds to the "flat plateau" of radiation in the low $p_{\perp}$ limit; it is also responsible for the "fan invariance" as follows from the exact relation

$$
\frac{d^{2} n}{d p_{\|} d \vartheta}=\frac{d^{2} n}{d \xi_{p} d p_{\perp}}
$$

with $\xi_{p}=\ln (P / p)$ defined in terms of the momentum $p$. The second term gives the energy dependent correction which vanishes in the soft limit $p_{\perp} \rightarrow$ $Q_{0}$; the cms energy $\sqrt{s}$ enters only through the variable $P_{\perp}=p_{\perp} / x$ with $x=2 E / \sqrt{s}$. The MLLA correction involves the exponent $G$ and its derivative

$$
G(\xi, Y)=\frac{a}{b} \ln \left(\frac{Y+\lambda}{\xi+\lambda}\right) \quad, \quad \frac{\partial}{\partial Y} G(\xi, Y)=\frac{a}{b(Y+\lambda)},
$$

so it again depends only on $Y+\lambda$ and $\xi$ (for soft particles one can assume $\xi \gg \lambda)$. Therefore our perturbative results fulfil the scaling law

$$
\frac{d^{2} n}{d \xi d \ln p_{\perp}}=F\left(\xi, p_{\perp}\right)
$$


i.e. the dependence on the cms energy enters only through the variable $\xi$ or $x$. In this way the violation of the "fan invariance" can be partly absorbed into the scaling variables $\xi$ or $x$.

The analytical predictions have been derived for the soft gluon radiation (with energies smaller than, say, $\mathcal{O}(1 \mathrm{GeV})$ ), while the angles are measured with respect to the primary parton axis. There are two effects which are beyond the perturbative calculations.

First, the direction of the primary parton is not directly known, but may be related to the jet axis or to the initial hadron or photon direction. Here the situation can be improved by measuring the energy-multiplicity correlation (2) taking the momentum of each particle in the event in turn as jet axis with the particle energy fraction as a weight. The angular correlation of this type has been studied using Monte Carlo events with favourable results 36.

Secondly, we can only perform calculations at the parton level, so in comparison with experiment an assumption on the effect of hadronization has to be made. This will be described in the next subsection.

\subsection{Relation between parton and hadron distributions}

According to the LPHD concept, we assume that parton and hadron spectra are proportional to each other if momenta are large compared to $Q_{0}$. This picture has so far only been applied to observables which are integrated over $p_{\perp}$, while we are now interested in distributions differential in $p_{\perp}$. Since perturbative formulae explicitly contain the cut-off $p_{\perp}>Q_{0}$, they cannot indeed be directly compared with the data at small $p_{\perp}$, but they have to be modified in order to provide a smooth behaviour in this region. There is no unique prescription to be followed in order to build this bridge between parton and hadron level, and what we are proposing here is a simple phenomenological ansatz which is consistent with kinematical requirements and the scaling behaviour in the soft limit.

In analogy to the relation (同) applied to the energy spectra in Section 2 , we take the transverse momentum cut-off $Q_{0}$ as an effective mass of the hadron with $E_{h}^{2}=p_{h}^{2}+Q_{0}^{2}$ and we then replace

$$
p_{\perp, p}^{2}=p_{\perp, h}^{2}+Q_{0}^{2}, \quad E_{p}=E_{h}
$$

in eqs. (16) and (17) on the r.h.s. as well as on the l.h.s. This choice guarantees the proper kinematical limits $p_{\perp, p} \geq Q_{0}$ and $p_{\perp, h} \geq 0$ for parton and hadron spectra, while they coincide for large $p_{\perp}$. Furthermore we use as

before $\left.E \frac{d n}{d p}\right|_{h}=\left.\frac{d n}{d \xi_{E}}\right|_{p}$. So for hadrons one can write (dropping the label $h$ 
everywhere)

$$
E \frac{d n}{d p d p_{\perp}}=\frac{p_{\perp}}{p_{\perp}^{2}+Q_{0}^{2}} F\left(\xi_{E}, \sqrt{p_{\perp}^{2}+Q_{0}^{2}}\right)
$$

with the function $F$ defined by (21) at the parton level, whereby the replacements (22) are applied. Once again, relations such as (22) are not an essential part of the perturbative LPHD picture, rather they should be considered as a plausible extension into the region $p_{\perp} \lesssim Q_{0}$, consistent with kinematics and scaling properties.

Combining now (23) and (19), one obtains quantitative theoretical predictions for the angular distribution of particles. Results for different values of $p_{\|}$at $\sqrt{s}=14 \mathrm{GeV}$ are shown in Fig. 6 for $Q_{0}=0.5 \mathrm{GeV}$ and $\lambda=0.05$. Choosing such parameters, the results describe reasonably well the experimental data obtained by the TASSO collaboration 19 in the region of low $p_{\|}$, while some deviation is visible for larger values of $p_{\|}$. The large values of $p_{\|}$and of angles correspond, however, to large values of the transverse momentum $p_{\perp}$, where our approximations are not valid any more. This point is particularly important for the study of "fan invariance", i.e., the energy independence of the renormalized angular distribution: the distributions presented by the TASSO collaboration 19] are normalized to unity in the full angular region and therefore not directly accessible by our calculations.

A better test of the scaling law (14) can be obtained from the direct study of the double differential distribution $d^{2} n / d p_{\|} d p_{\perp}$ in absolute normalization (i.e., normalized to the particle multiplicity) as a function of the transverse momentum $p_{\perp}$ at fixed $p_{\|}$. Fig. 7 shows the theoretical predictions in MLLA with the same parameters as in Fig. 6 at $p_{\|}=0.8 \mathrm{GeV}$ at four different values of $\mathrm{cms}$ energy $\sqrt{\mathrm{s}}$. A good scaling behaviour is visible in the region of very low transverse momentum, whereas the position of the peak and the tail are found to smoothly depend on the cms energy. If the distributions were rescaled to the same area as in [19], the overall energy dependence would be rather small.

In order to better understand the origin of this energy dependence, it is useful to separately look at the contribution of the different terms in the theoretical formula. This is shown in Fig. 8, where the $d^{2} n / d p_{\|} d p_{\perp}$ distributions at $p_{\|}=0.8 \mathrm{GeV}$ and $\sqrt{s}=34 \mathrm{GeV}$ are shown for different approximations: the DLA (Born term only), DLA (Born plus next order term) and MLLA.

\footnotetext{
${ }^{6}$ This value for $Q_{0}$ is largely determined by the position of the maximum in the angular distribution and is larger than the value taken above for the energy spectrum. A unification is possible in principle by calculating the energy spectrum from the integration over the modified double differential spectrum $d^{2} n / d E d p_{\perp}$, but this is not attempted here.
} 
Whereas the Born term of DLA, which is independent of cms energy, is dominant at small $p_{\perp}$, the other terms become important with increasing $p_{\perp}$ and give rise to the energy dependence visible in Fig. 7. As the next order term becomes important for larger $p_{\perp}$, we also compare in Fig. 8 with the prediction from the Limiting Spectrum which corresponds to an all order summation within a high energy approximation (taking the same $Q_{0}, \lambda=$ 0 and normalization $\left(K_{h}=3.5\right)$ adjusted to match the MLLA result). It is remarkable how close are the predictions from the MLLA and from the Limiting Spectrum. This result suggests that retaining only the first two terms in the DLA solution gives indeed a reasonable approximation in the region of low transverse momentum.

The deviation from the scaling behaviour (14) is due to the weak logarithmic dependence of the correction terms on the primary energy. As a consequence of (23) with $p \sim p_{\|}$the invariant density, after our modification (22), still follows the scaling behaviour

$$
E \frac{d n}{d^{3} p}=f\left(x_{E}, p_{\perp}\right)
$$

i.e., for fixed $p_{\perp}$ it depends on particle and $\mathrm{cms}$ energies only through $x_{E}=$ $2 E / \sqrt{s}$. This scaling behaviour (24) is expected to hold in a wider kinematic region than eq. (14).

\section{Further tests of the perturbatively-based picture of soft particle production}

\subsection{Sensitivity to the colour charge of primary partons}

The nearly energy independent soft end of the particle spectrum has been interpreted above as a consequence of the coherent soft gluon emission together with the LPHD. Alternatively, one may argue that at these low momenta of few hundred $\mathrm{MeV}$ we observe the purely hadronic phenomena not related to the perturbative QCD at all. It is therefore important to test further the perturbative nature of the soft particle production.

The production rate in the limit (6) cannot be predicted in absolute terms, but we note the dependence of this rate on the colour charge factors $C_{F}$ and $N_{C}$ for the jets originating from a primary quark or gluon respectively. Therefore, we have argued in [15] that the sensitivity of the soft end of the spectrum to the colour of the primarily produced hard partons provides a crucial test of the perturbatively based picture and can teach us about the region of its validity. 
The prediction for the ratio of multiplicities in gluon and quark jets $R_{g / q}=$ $N_{C} / C_{F}=2.25$ has been made long ago [39] but holds only at asymptotic energies. At existing energies this ratio is measured to be smaller but not in disagreement with the perturbative QCD analysis (see, for example [3, 40,17]). Our present discussion concerns only the soft particles: in this kinematic regime the asymptotic predictions on the spectrum, in particular the scaling properties, are well satisfied and therefore the asymptotic ratio $R_{g / q}$ in this limit could be asymptotic too without contradicting the global results.

Therefore, in order to test this scenario, one would like to compare the soft particle production in $q \bar{q}$ and $g g$ events. Whereas there are well measured spectra down to small momenta of about $200 \mathrm{MeV}$ from quark jets in $e^{+} e^{-}$and DIS processes (see Figs. 1 and 2) the direct production of a $g g$ final state is more difficult.] To perform such a comparison nevertheless we have discussed in [15 two different approaches.

First, there is the comparatative study of two jet events arising from reactions with primary hadrons and photons mediated by quark or gluon exchange processes. If the exchange is sufficiently hard the two types of processes yield primary colour sources of the desired $q \bar{q}$ and $g g$ types. We will dicuss below how these limits can be derived explicitely from the hard scatterings in the limit of small momentum transfer. The phenomenological studies along this line are described in papers [15].

Secondly, one may consider final states with more than two partons. In certain collinear limits such final states approach a $q \bar{q}$ or $g g$ type antennae. A simple example is provided by the process $e^{+} e^{-} \rightarrow q \bar{q} g$ which for the configurations with angle $\Theta_{q g} \sim 0$ or $\Theta_{\bar{q} g} \sim 0$ behaves like a $q \bar{q}$ and for $\Theta_{\bar{q} q} \sim 0$ behaves like a $g g$ final state [42]. Such extreme configurations are in general not realistic. However, we can study the radiation perpendicular to the event plane (say, in the cone with not too large opening angle $\delta$ ), then the particle density at low momenta should vary by the factor $N_{C} / C_{F}$ when going from the $q \bar{q}$ to the $g g$ type configuration and this variation can be predicted also for the intermediate kinematical range. Similarly, one can study such an associated perpendicular radiation in parton-parton scattering processes with quark or gluon exchange at any angle (see Fig. 9).

In the following we will present the expectations for various processes; the main results for $e^{+} e^{-} \rightarrow q \bar{q} g$ have been given already in [15].

\footnotetext{
${ }^{7}$ At high energy $e^{+} e^{-}$colliders the process $\gamma \gamma \rightarrow g g$ may become a prospective source of clear information 41
} 


\subsection{Soft radiation in $e^{+} e^{-} \rightarrow q \bar{q} g$}

It was realized long ago that the overall structure of soft particle angular distribution in multijet events in hard processes is governed by the underlying dynamics of colour at small distances. An instructive known example is the perturbative explanation 43 of the particle angular flow in $e^{+} e^{-} \rightarrow q \bar{q} g$ events ("string effect" 44). The multiplicity flows build up a colour portrait of an event which can be used as a natural "partonometer" mapping the primary interaction short-distance process 40, 38, 2]. In these studies the drag effect on the particles within the production plane is caused by the primary colour dipoles. More recently, the azimuthal angular distribution around the jet directions [50,51] have been studied. The partonometry ideas are strongly supported by a wealth of experimental data from $e^{+} e^{-} \rightarrow q \bar{q} g$ annihilation (see e.g. [3]) and the TEVATRON $p \bar{p}$ collider [10].

In the present application we are interested in the particle production at low momenta $p$ of order $Q_{0}$. A straightforward calculation is possible for the radiation perpendicular to the production plane of the jets. Otherwise, in multijet events, the cut-off condition $p_{\perp}>Q_{0}$ is more difficult to implement. Also, in this case $p_{\perp}$ appears as argument of $\alpha_{s}$ and does not change for boosts of partons within the plane. In the collinear limits for the 3 -jet events one obtains the radiation transverse to the jet for which the coherence arguments apply in the first place.

In the simplest case of a boosted $q \bar{q}$ pair, or equivalently, in $e^{+} e^{-} \rightarrow q \bar{q} \gamma$ the radiation pattern of gluons with momentum $p$ in direction $\vec{n}$ is given by 43

$$
\begin{aligned}
\frac{d N_{q \bar{q}}}{d \Omega_{\vec{n}} d p} & =\frac{\alpha_{s}}{(2 \pi)^{2} p} W^{q \bar{q}}(\vec{n}) \\
W^{q \bar{q}}(\vec{n}) & =2 C_{F}(\widehat{i j}), \quad(\widehat{i j})=\frac{a_{i j}}{a_{i} a_{j}}
\end{aligned}
$$

where $a_{i j}=\left(1-\vec{n}_{i} \vec{n}_{j}\right)$ and $a_{i}=\left(1-\vec{n} \vec{n}_{i}\right)$ for $q$ and $\bar{q}$ in direction $\vec{n}_{i}$ and $\vec{n}_{j}$. For the radiation perpendicular to the plane we simply obtain

$$
(\widehat{i j})=1-\cos \Theta_{i j}
$$

with the relative angle $\Theta_{i j}$ between the primary partons. This yields the two limits $(\widehat{i j})=0,2$ for parallel and antiparallel momenta respectively.

As we are interested only in the soft particles and the relative variations between different processes or configurations we restrict ourselves to the single gluon bremsstrahlung of order $\alpha_{s}$ as in (25). The MLLA corrections will become important for larger momenta (see e.g. the discussion in 43,15). 
The soft gluon radiation in the $q \bar{q} g$ process is given by the antenna pattern (defined as in (25))

$$
W^{q \bar{q} g}(\vec{n})=N_{C}\left[(\widehat{1+})+(\widehat{1-})-\frac{1}{N_{C}^{2}}(\widehat{+-})\right]
$$

where $(+,-, 1)$ refer to $(q, \bar{q}, g)$ and this bremsstrahlung pattern holds, irrespective of whether the partons are incoming or outgoing. With (27) one can obtain the particle density in the direction perpendicular to the production plane.

It is convenient to normalize the production rate of the soft particles to the corresponding rate in the standard process $e^{+} e^{-} \rightarrow q \bar{q}$ in its rest frame with $W_{\perp}^{q \bar{q}}=4 C_{F}$ according to (26) and define the ratio

$$
R_{\perp}^{p}(p) \equiv \frac{d N_{\perp}^{p} / d \Omega_{\vec{n}} d p}{d N_{\perp}^{q \bar{q}} / d \Omega_{\vec{n}} d p}=\frac{W_{\perp}^{p}}{4 C_{F}} .
$$

for a general process $p$. For the perpendicular radiation in the 3 jet events one finds

$$
R_{\perp}^{q \bar{q} g}(p)=\frac{N_{C}}{4 C_{F}}\left[2-\cos \Theta_{1+}-\cos \Theta_{1-}-\frac{1}{N_{C}^{2}}\left(1-\cos \Theta_{+-}\right)\right] .
$$

In our approximation the momentum spectrum does not depend on the angles $\Theta_{i j}$ between the jets. It will be interesting to study the spectrum $d n / d^{2} p$ in such a cone experimentally and to find out whether the angular dependence of the particle density in (30) holds down to small momenta.

It is also interesting to note the difference of this prediction to the large $N_{C}$ approximation in which the $q \bar{q} g$ event is treated as a superposition of two $q \bar{q}$ dipoles (see, e.g., 44]). In this case the last term in eq. (30) drops out and $C_{F}=\left(N_{C}^{2}-1\right) / 2 N_{C} \simeq N_{C} / 2$. This yields

$$
R_{\perp} \simeq \frac{1}{2}\left[2-\cos \Theta_{1+}-\cos \Theta_{1-}\right] \quad\left(\text { large } N_{C}\right)
$$

Predictions from these formulae for $R_{\perp}$ are presented in Table 1 for various relative angles $\Theta_{i j}$. Note, in particular, the limiting case $R_{\perp}=1$ for soft or collinear primary gluon emission and the proper $g g$ limit for the parallel $q \bar{q}$ $\left(\Theta_{+-}=0\right)$ configuration, as expected.

The role of the large- $N_{C}$ limit can be investigated also by studying the production rate in 3-jet events normalized to the sum of rates from the corresponding 2-jet events (dipoles) with opening angle $\Theta_{1+}$ and $\Theta_{1-}$ respectively:

$$
\begin{aligned}
\tilde{R}_{\perp} & \equiv \frac{d N_{\perp}^{q \bar{q} g}}{d N_{\perp}^{q \bar{q}}\left(\Theta_{1+}\right)+d N_{\perp}^{q \bar{q}}\left(\Theta_{1-}\right)} \\
& =\frac{N_{C}^{2}}{N_{C}^{2}-1}\left(1-\frac{1}{N_{C}^{2}} \frac{1-\cos \left(\Theta_{1+}+\Theta_{1-}\right)}{2-\cos \Theta_{1+}-\cos \Theta_{1-}}\right)
\end{aligned}
$$




\begin{tabular}{||c|c|c||}
\hline & $R_{\perp}$ & $R_{\perp}$ (large $\left.N_{C}\right)$ \\
\hline$\Theta_{1+}=\pi-\Theta_{1-}$ & 1 & 1 \\
(collinear or soft gluons) & & 1.18 \\
$\Theta_{1+}=\Theta_{+-}=\frac{5}{6} \pi$ & 1.21 & 1.35 \\
$\Theta_{1+}=\Theta_{+-}=\frac{3}{4} \pi$ & 1.42 & 1.5 \\
$\Theta_{1+}=\Theta_{1-}=\frac{2}{3} \pi$ & 1.59 & \\
$($ Mercedes $)$ & & 2 \\
$\Theta_{1+}=\Theta_{1-}=\pi$ & $\frac{N_{C}}{C_{F}}=2.25$ & \\
$(q \bar{q}$ antiparallel to $g)$ & & \\
\hline
\end{tabular}

Table 1: Prediction for the ratio $R_{\perp}=d N_{\perp}^{q \bar{q} g} / d N_{\perp}^{q \bar{q}}$ from (30) and its large$N_{C}$-approximation (31) for different configurations of the $q \bar{q} g$ events $\left(\Theta_{1+} \equiv\right.$ $\left.\Theta_{g q}, \Theta_{1-} \equiv \Theta_{g \bar{q}}, \Theta_{+-}=2 \pi-\Theta_{1+}-\Theta_{1-}\right)$.

This ratio measures directly the deviation from the large- $N_{C}$ limit $\tilde{R}_{\perp}=1$ and thereby from the $q \bar{q}$-dipole approximation. This approximation is not necessarily limited towards soft particle production. For the simple case of Mercedes-like events $\left(\Theta_{1+}=\Theta_{1-}=\Theta_{+-}\right)$one obtains $\tilde{R}_{\perp}=17 / 16=1.06$. The 2-jet rates for relative angle $\Theta_{i j}$ which appear in the denominator of eq. (32) could be found experimentally from the corresponding $q \bar{q} \gamma$ final states.

Let us list a few further results:

a) A particularly simple situation is met for Mercedes-type events where no jet identification is necessary for the above measurements.

b) The large angle radiation is independent of the mass of the quark (for $\left.\Theta \gg m_{Q} / E_{\text {jet }}\right)$.

c) The above predictions for the ratios $R_{\perp}, \tilde{R}_{\perp}$ are derived for the soft particles according to the bremsstrahlung formula (25). One may also consider the particle flow integrated over momentum, as in the discussion of the string/drag effect. In this case one has to include all higher order contributions which take into account the fact that the soft gluon is part of a jet generated from a primary parton. Then the angular flow $d N / d \Omega_{\vec{n}}$ is given by the product of the radiation factor $W(\vec{n})$ and a "cascading factor" [43]. For the ratio of multiplicity flows one obtains the same predictions (30) and (32) as the cascading factors cancel. It will be interesting to see to what extent 
the predicted angular dependence for both quantities - the multiplicity flows and the soft particle yields - are satisfied experimentally.

d) The similarity of particle flows in 3-jet and 2-jet events with corresponding angles $\Theta_{1+}, \Theta_{1-}$, as expected in the large- $N_{C}$ approximation, should also apply to further details of the final state such as the particle ratios. Since the Lorentz transformation along the boost direction produces a larger drag for heavier particles, one may expect that after the boost the $K / \pi$ and $p / \pi$ ratios for soft particles decrease and then the same is true for soft particles in 3 -jet events in comparison to 2 -jet events in their rest frame 9 .

\subsection{Soft radiation accompagnying photo-production of di-jets}

\subsubsection{Direct and resolved processes}

Next we consider the photoproduction of di-jets. The soft bremsstrahlung depends only on the momentum vectors of the partons but not on the virtuality of the photon. Our results can be applied to $\gamma p$ as well as to $\gamma \gamma$ collisions. The di-jet photoproduction has been studied recently in detail at HERA 46,47. In di-jet production one can distinguish in the leading order QCD approach the direct and the resolved processes 488. In the first case the photon participates directly in the hard scattering subprocess and transfers a large fraction $\left(x_{\gamma} \sim 1\right)$ of its primary energy to the secondary jets; in the second case, the hard scattering subprocess involves the partons in the protons and also in the photons and the energy fraction is smaller $\left(x_{\gamma}<1\right)$. The data indeed show two peaks in the distribution of $x_{\gamma}$ corresponding to the two types of processes.

The direct processes are mediated by

$$
\begin{aligned}
& \gamma\left(p_{1}\right)+g\left(p_{2}\right) \rightarrow q\left(p_{3}\right)+\bar{q}\left(p_{4}\right) \\
& \text { b) } \gamma\left(p_{1}\right)+q\left(p_{2}\right) \rightarrow q\left(p_{3}\right)+g\left(p_{4}\right),
\end{aligned}
$$

the so-called photon-gluon-fusion and QCD-Compton processes respectively. Their differential cross sections are given by

$$
\begin{aligned}
\frac{d \sigma^{a}}{d t} & =\frac{\pi \alpha \alpha_{s} Q_{q}^{2}}{s^{2}}\left(\frac{u}{t}+\frac{t}{u}\right) \\
\frac{d \sigma^{b}}{d t} & =\frac{8 \pi \alpha \alpha_{s} Q_{q}^{2}}{3 s^{2}}\left(-\frac{t}{s}+-\frac{s}{t}\right)
\end{aligned}
$$

${ }^{8}$ This expectation has been verified in the JETSET Monte Carlo 45. We thank T. Sjöstrand for providing us with this information. 
where $Q_{q}$ is the quark charge and

$$
\begin{gathered}
s=\left(p_{1}+p_{2}\right)^{2}, \quad t=\left(p_{1}-p_{3}\right)^{2} \text { and } u=\left(p_{1}-p_{4}\right)^{2} \\
t=-s\left(1-\cos \Theta_{s}\right) / 2 \text { and } u=-s\left(1+\cos \Theta_{s}\right) / 2
\end{gathered}
$$

with the scattering angle $\Theta_{s}$ in the di-jet $\mathrm{cms}$. The cross section is maximal for small $t$ (or $u$ ) where it is dominated by quark exchange.

In the resolved processes the partons $(q, \bar{q}$ and $g)$ in the photon interact with the partons of the proton. We restrict ourselves in this discussion to the small angle scattering where the gluon exchange contribution dominates and consider the subprocesses

$$
\begin{array}{lll}
\text { c) } & g\left(p_{1}\right)+g\left(p_{2}\right) & \rightarrow g\left(p_{3}\right)+g\left(p_{4}\right) \\
\text { d) } & g\left(p_{1}\right)+q\left(p_{2}\right) & \rightarrow g\left(p_{3}\right)+q\left(p_{4}\right) \\
e) & q\left(p_{1}\right)+q^{\prime}\left(p_{2}\right) & \rightarrow q\left(p_{3}\right)+q^{\prime}\left(p_{4}\right) \\
f) & q\left(p_{1}\right)+q\left(p_{2}\right) & \rightarrow q\left(p_{3}\right)+q\left(p_{4}\right) \\
g) & q\left(p_{1}\right)+\bar{q}\left(p_{2}\right) & \rightarrow q\left(p_{3}\right)+\bar{q}\left(p_{4}\right)
\end{array}
$$

(in processes $d$ and $f$ the $q$ 's could be replaced by the $\bar{q}$ 's).

The different exchanges in the direct and resolved processes cause different distributions $d n / d t \sim t^{-1}$ and $d n / d t \sim t^{-2}$ respectively and this difference is clearly seen in the data [46]. Therefore in this case, by choosing suitable intervals of $x_{\gamma}$, one can select processes of different colour structure and one can check whether or in which kinematical interval the low momentum particles are sensitive to it. To this end we calculate the respective production rates for the soft particles.

For the given total energy the hard process depends on 3 variables which we choose here for simplicity as the incoming parton energy fractions $x_{1}$ and $x_{2}$ and the momentum transfer $t$. The cross section for producing the di-jets with and without accompagnying soft gluons is then given by

$$
\begin{aligned}
\frac{d \sigma}{d x_{1} d x_{2} d t d \Omega_{\vec{n}} d p} & =\sum_{i j k l} f_{i}\left(x_{1}, \mu^{2}\right) f_{j}\left(x_{2}, \mu^{2}\right) \frac{d \sigma^{i j \rightarrow k l}}{d t} \frac{\alpha_{s}}{(2 \pi)^{2} p} W^{i j ; k l}(\vec{n}) \\
\frac{d \sigma}{d x_{1} d x_{2} d t} & =\sum_{i j k l} f_{i}\left(x_{1}, \mu^{2}\right) f_{j}\left(x_{2}, \mu^{2}\right) \frac{d \sigma^{i j \rightarrow k l}}{d t}
\end{aligned}
$$

where $f_{i}\left(x, \mu^{2}\right)$ denotes the parton structure functions at scale $\mu^{2}$. We are interested in the density of the soft radiation, i.e. in the ratio

$$
\frac{d N}{d \Omega_{\vec{n}} d p}=\frac{d \sigma}{d x_{1} d x_{2} d t d \Omega_{\vec{n}} d p} / \frac{d \sigma}{d x_{1} d x_{2} d t}
$$


where often in practical applications an integration over ranges of the kinematical variables $x_{1}, x_{2}$ and $t$ has to be performed. If only one subprocess $[i j \rightarrow k l]$ contributes we come back to relations analogous to (25).

\subsubsection{Soft particles in direct processes}

The angular factors required in (44) for the partons $q, \bar{q}$ and $g$ involved in processes a) and b) in (33) and (34) are given by (28) with the proper relabeling of the partons (see also [52])

$$
\begin{aligned}
W^{a}(\vec{n}) & =N_{C}\left[(\widehat{23})+(\widehat{24})-\frac{1}{N_{C}^{2}}(\widehat{34})\right], \\
W^{b}(\vec{n}) & =N_{C}\left[(\widehat{34})+(\widehat{24})-\frac{1}{N_{C}^{2}}(\widehat{23})\right] .
\end{aligned}
$$

For the case of perpendicular radiation in the di-jet rest frame we have simply

$$
(\widehat{23})=(\widehat{14})=1+\cos \Theta_{s}, \quad(\widehat{24})=(\widehat{13})=1-\cos \Theta_{s}, \quad(\widehat{12})=(\widehat{34})=2 .
$$

If either of the two direct processes a) or b) dominates we obtain for the respective rates, normalized as in (29)

$$
\begin{aligned}
R_{\perp}^{a} & =1, \\
R_{\perp}^{b} & =\frac{N_{C}}{4 C_{F}}\left[3-\cos \Theta_{s}-\frac{1}{N_{C}^{2}}\left(1+\cos \Theta_{s}\right)\right] .
\end{aligned}
$$

This means that the soft radiation in the photon gluon fusion process is the same as in the standard process $e^{+} e^{-} \rightarrow q \bar{q}$ irrespectively of the scattering angle $\Theta_{s}$. In case of the QCD-Compton process we find in the limit of small angles $\Theta_{s} \ll 1$ which is dominated by quark exchange $R_{\perp}^{b}=1$. In the case of backward scattering with $\Theta_{s} \sim \pi$ (i.e. the transition $\gamma \rightarrow g$ at small angle) we have effectively a colour octet channel and find $R_{\perp}^{b}=N_{C} / C_{F}$ as in case of a $g g$ final state. In the large $N_{C}$ approximation we can consider the bremsstrahlung in the quark exchange processes as coming from a single colour dipole whereby the second is inactive (for $\Theta_{s}=0$ ); in case of octet exchange the radiation comes from two dipoles and adds incoherently.

If the two jets are not identified one has to add the cross sections for scatterings at angles $\Theta_{s}$ and $\pi-\Theta_{s}$. One finds

$$
\begin{aligned}
& R_{\perp}^{a, \text { sym }}=1 \\
& R_{\perp}^{b, \text { sym }}=\frac{N_{C}}{2 C_{F}} \frac{G(x)\left[1+x-y / N_{C}^{2}\right]+G(y)\left[1+y-x / N_{C}^{2}\right]}{G(x)+G(y)}
\end{aligned}
$$


with $x=-t / s, y=-u / s$ and $G(z)=z+z^{-1}$. So the latter ratio rises from $R_{\perp}^{b, s y m}=1$ at $\Theta_{s}=0$ to $R_{\perp}^{b, s y m}=\frac{13}{8}=1.63$ at $\Theta_{s}=\frac{\pi}{2}$. Therefore, in the kinematic region where one of the processes dominates one can check the variation of the soft particle density with scattering angle $\Theta_{s}$, analogously to the case of $e^{+} e^{-} \rightarrow q \bar{q} g$. In the more general situation one has to use the superposition (44) with the structure functions as weights.

In the further discussion we restrict ourselves to the scattering at small angles where quark or gluon exchanges dominate to simplify the discussion. Then in both processes a) and b) the quark exchange with $R_{\perp}^{b}=1$ dominates and this result also holds in a general superposition (44) of symmetrized direct processes.

\subsubsection{Soft production from resolved processes}

For small angles $-t \ll s$ the expressions for the radiation patterns simplify significantly [38. The antenna patterns accompagnying the $2 \rightarrow 2$ parton scattering through the t-channel exchange are then given by

$$
\begin{aligned}
W^{c}(\vec{n})= & N_{C}\left[(\widehat{13})+(\widehat{24})+\frac{1}{2}\{(\widehat{12})+(\widehat{34})+(\widehat{14})+(\widehat{23})\}\right], \\
W^{d}(\vec{n})= & N_{C}\left[(\widehat{13})-\frac{1}{N_{C}^{2}}(\widehat{24})+\frac{1}{2}\{(\widehat{12})+(\widehat{34})+(\widehat{14})+(\widehat{23})\}\right], \\
W^{e}(\vec{n})= & W^{f}(\vec{n})=2 C_{F}[(\widehat{14})+(\widehat{23}) \\
& \left.+\frac{1}{2 N_{C} C_{F}}\{2((\widehat{12})+(\widehat{34}))-(\widehat{14})-(\widehat{23})-(\widehat{13})-(\widehat{24})\}\right], \\
W^{g}(\vec{n})= & 2 C_{F}[(\widehat{12})+(\widehat{34}) \\
& \left.+\frac{1}{2 N_{C} C_{F}}\{2((\widehat{14})+(\widehat{23}))-(\widehat{12})-(\widehat{43})-(\widehat{13})-(\widehat{24})\}\right],
\end{aligned}
$$

Considering the subprocesses $i=c, \ldots, g$ separately we obtain the ratios

$$
\begin{aligned}
R_{\perp}^{c} & =\frac{N_{C}}{4 C_{F}}\left(5-\cos \Theta_{s}\right), \\
R_{\perp}^{d} & =\frac{N_{C}}{4 C_{F}}\left[4-\frac{1}{N_{C}^{2}}\left(1-\cos \Theta_{s}\right)\right], \\
R_{\perp}^{e, f} & =\left(1+\cos \Theta_{s}\right)+\frac{1}{N_{C} C_{F}}, \\
R_{\perp}^{g} & =2+\frac{1}{2 N_{C} C_{F}}\left(3 \cos \Theta_{s}-1\right) .
\end{aligned}
$$


At low scattering angles $\Theta_{s} \ll 1$ where the gluon exchange dominates all these ratios $R_{\perp}^{i}$ are

$$
R_{\perp}^{i}=\frac{N_{C}}{C_{F}} \approx 2.25 \quad(i=c, \ldots, f)
$$

and for the important processes c) and d) they vary only slowly with angle. Therefore also for the superposition (44) of all processes the ratio will be close to (62) for not too large angles. On the other hand, these ratios differ markedly (by about a factor of 2) from $R_{\perp}^{a, b}$ for the direct photoproduction. In the large $N_{C}$ limit and at $\Theta_{s} \ll 1 R_{\perp}^{c-g}$ correspond to the incoherent sum of two $q \bar{q}$ antennae-dipoles, while $R_{\perp}^{a, b}$ are given by only one $q \bar{q}$ antenna.

Therefore, when comparing the perpendicular radiation pattern in direct and resolved photoproduction of di-jets one expects an increased particle density in the latter case by about a factor of 2 . It will be interesting to find out experimentally whether such a difference occurs already at such low momenta as a few hundred $\mathrm{MeV}$. Such a finding would provide an important support for the relevance of perturbative QCD, in particular of the colour coherence, to the soft particle production.

\subsection{Hard processes in hadron hadron collisions}

The tests which are interesting from our point of view are very similar to those discussed for photoproduction in the last subsection. First there are the subprocesses (39)-(42) of parton parton collisions (at small angles) which we discussed in connection with resolved photoproduction. Secondly, there are the processes with a final state direct photon (or, equivalently a weak vector boson $\mathrm{W}$ or $\mathrm{Z}$ ) produced together with a hadronic jet with opposite transverse momenta (see, e.g. [2,51]). These processes correspond to the direct processes in photoproduction. The relevant subprocesses are

$$
\begin{aligned}
& \left.a^{\prime}\right) \quad q\left(p_{1}\right)+\bar{q}\left(p_{2}\right) \rightarrow \gamma\left(p_{3}\right)+g\left(p_{4}\right), \\
& \left.b^{\prime}\right) \quad q\left(p_{1}\right)+g\left(p_{2}\right) \rightarrow \gamma\left(p_{3}\right)+\bar{q}\left(p_{4}\right)
\end{aligned}
$$

and the soft bremsstrahlung is again found from (28) with appropriate relabeling

$$
\begin{aligned}
W^{a^{\prime}}(\vec{n}) & =N_{C}\left[(\widehat{14})+(\widehat{24})-\frac{1}{N_{C}^{2}}(\widehat{12})\right] \\
W^{b^{\prime}}(\vec{n}) & =N_{C}\left[(\widehat{12})+(\widehat{24})-\frac{1}{N_{C}^{2}}(\widehat{14})\right] .
\end{aligned}
$$


Then we obtain for the perpendicular radiation

$$
\begin{aligned}
R_{\perp}^{a^{\prime}} & =1, \\
R_{\perp}^{b^{\prime}} & =\frac{N_{C}}{4 C_{F}}\left[3-\cos \Theta_{s}-\frac{1}{N_{C}^{2}}\left(1+\cos \Theta_{s}\right)\right]
\end{aligned}
$$

just as in case of direct photoproduction.

There is an interesting difference with the case of photoproduction. At high primary energies the parton collisions with initial gluons are more likely. Therefore in photoproduction the photon-gluon fusion process (a) without angular dependence in the soft particle production rate is favoured whereas at the hadron collider the other process $\left(b^{\prime}\right)$ with angular dependence is more likely. This offers the possibility to study the angular dependence of the soft particle production in the same process.

\section{Conclusions}

The analytical perturbative approach to multiparticle production together with the assumption of LPHD has proven to be very successful in the description of various inclusive characteristics of jets. It is of importance to investigate further the potential and limitations of this picture, in particular in the soft region where hadronization effects could in principle wash out the perturbative predictions.

The soft particles follow a striking prediction of the perturbative analysis. The particle density at low momentum is nearly energy independent over two orders of magnitude in the cms energy of $e^{+} e^{-}$annihilation and this behaviour is supported now also by the data from ep collisions. More generally, the analytic calculations suggest a scaling law for particles at low transverse momentum; such a property was observed already long ago in the PETRA energy range. The scaling law is derived analytically for soft gluons which are emitted coherently from all other more energetic colour sources. In the perturbative description this process of single gluon emission is dominated by the Born term of $\mathcal{O}\left(\alpha_{s}\right)$ which is energy independent. At larger $p_{\perp}$ gluons are more frequently produced through cascading processes of higher order in $\alpha_{s}$. Remarkably, the hadrons follow this prediction demonstrating the relevance of LPHD also for soft particles.

It is very important to further clarify whether the observed scaling behaviour is just a general property of hadronization or should be considered as consequence of the perturbative QCD as in our picture. In the latter case the density of the low momentum particles should vary in a well defined

way with the type of reactions and orientations of the primary partons in the 
hard process. In this paper we derive predictions for processes for which data are already available. Experimental studies of the proposed type would clarify at which scale of the low transverse momentum or energy the behaviour expected from the perturbative analysis sets in.

\section{Acknowledgements}

We would like to thank A. De Angelis, Yu.L. Dokshitzer, D. Kant, P. Mättig, R. Orava, G. Thompson, T. Sjöstrand and E.A. De Wolf for useful discussions. 


\section{References}

[1] A. Bassetto, M. Ciafaloni and G. Marchesini, Phys. Rep., C100 (1983) 201.

[2] Yu. L. Dokshitzer, V. A. Khoze, A. H. Mueller and S. I. Troyan, Rev. Mod. Phys., 60 (1988) 373; "Basics of Perturbative QCD", ed. J. Tran Thanh Van, Editions Frontiéres, Gif-sur-Yvette, 1991.

[3] for a recent phenomenological status of the analytical perturbative approach, see V.A. Khoze and W. Ochs, Int. J. Mod. Phys. A12 (1997) 2949.

[4] Yu. L. Dokshitzer and S. I. Troyan, Proc. 19th Winter School of the LNPI, Vol. 1, p.144; Leningrad preprint LNPI-922 (1984).

[5] A. H. Mueller, Nucl. Phys., B213 (1983) 85; erratum quoted ibid., B241 (1984) 141.

[6] Ya. I. Azimov, Yu. L. Dokshitzer, V. A. Khoze and S. I. Troyan, Z. Phys., C27 (1985) 65 and C31 (1986) 213.

[7] H1 Coll., S. Aid et al., Nucl. Phys. B445 (1995) 3.

[8] H1 Coll., C. Adloff et al., Nucl. Phys. B504 (1997) 3.

[9] ZEUS Coll., M. Derrick et al., Z. Phys. 67 (1995) 93.

[10] CDF Coll., F. Abe et al., Phys. Rev. D50 (1994) 5562;

D0 Coll., B. Abbott et al., hep-ex/9706012, to be published in Phys. Rev. Lett.;

D0 Coll., D.E. Cullen-Vidal, FERMILAB preprint, FERMILAB Conf96-304-EI (1996).

[11] CDF Coll., A. Korytov, in Proc. of QCD '96, (Montpellier, France, 1996), Eds. S. Narison, Nucl. Phys. B (Proc. Suppl.) 54A (1997) 67.

[12] Yu. L. Dokshitzer, V. S. Fadin and V. A. Khoze, Phys. Lett., 115B (1982) 242; Z. Phys., C15 (1982) 325.

[13] A. Bassetto, M. Ciafaloni, G. Marchesini and A. H. Mueller, Nucl. Phys., B207 (1982) 189.

[14] S. Lupia and W. Ochs, Phys. Lett. B365 (1996) 339;

MPI-PhT/97-26, hep-ph/9704319, April 1997, Z. Phys. C in press. 
[15] V. A. Khoze, S. Lupia and W. Ochs, Phys. Lett. B394 (1997) 179;

Proc. 7th Int. Workshop on Multiparticle Production, Eds. R.C. Hwa, W. Kittel, W.J. Metzger and D.J. Schotanus, June 1966, Nijmegen, The Netherlands, World Scientific (1997), p. 358 (hep-ph/9610348).

[16] S. Lupia, in Proc. of QCD '96, (Montpellier, France, 1996), Eds. S. Narison, Nucl. Phys. B (Proc. Suppl.) 54A (1997) 55;

W. Ochs, in Proc. XXXVI Cracow School of Theor. Phys., (Zakopane, Poland, 1996), Acta Phys. Pol. B27 (1996) 3505.

[17] S. Lupia and W. Ochs, MPI-PhT/97-46, hep-ph/9707393, July 1997, Phys. Lett. B in press.

[18] V. A. Khoze, in in " $Z^{0}$ Physics", Cargèse 1990, Eds. M. Lévy, J-L. Basdevant, M. Jacob, D. Speiser, J. Weyers and R. Gastmans, NATO ASI, Series B: Physics Vol. 261, p. 419.

[19] TASSO Coll., M. Althoff et al., Z. Phys. C22 (1984) 307.

[20] B.L. Ioffe, V.A. Khoze and L.N. Lipatov, Hard Processes, Vol. 1, North Holland Publ., Amsterdam (1984).

[21] Yu. L. Dokshitzer, V. A. Khoze and S. I. Troyan, Int. J. Mod. Phys. A7 (1992) 1875.

[22] Yu. L. Dokshitzer, V. A. Khoze and S. I. Troyan, Z. Phys., C55 (1992) 107.

[23] DELPHI Coll., P. Abreu et al., Phys. Lett. B347 (1995) 447.

[24] TASSO Coll., W. Braunschweig et al., Z. Phys. C47 (1990) 187.

[25] OPAL Coll., M. Z. Akrawy et al., Phys. Lett., B247 (1990) 617.

[26] MARK I Coll., J. L. Siegrist et al., Phys. Rev. D26 (1982) 969.

[27] TOPAZ Coll., R. Itoh et al., Phys. Lett. B345 (1995) 335.

[28] ALEPH Coll., D. Buskulic et al., Z. Phys. C73 (1997) 409

[29] ARGUS Coll., S. Behrends et al., Phys. Rev. D31 (1985) 2161; TPC/2 $\gamma$ Coll., H. Aihara et al., Phys. Rev. Lett. 61 (1988) 1263;

[30] OPAL Coll., R. Akers et al., Z. Phys. C63 (1994) 181. 
[31] ALEPH Coll., R. Barate et a;., preprint CERN/PPE 96-186, to appear in Phys. Rep.

[32] MARK II Coll., J. F. Patrick et al., Phys. Rev. Lett. 49 (1982) 1232;

[33] L3 Coll., B. Adeva et al., Phys. Lett. B259 (1991) 199;

DELPHI Coll., P. Abreu et al., Phys. Lett. B372 (1996) 172;

OPAL Coll., M. Z. Akrawy et al.,Z. Phys. C72 (1996) 191;

OPAL Coll., K. Ackerstaff et al., preprint CERN-PPE/97-015, to appear in Z. Phys. C

[34] TASSO Coll., R. Brandelik et al., Phys. Lett. B94 (1980) 444;

TASSO Coll., W. Braunschweig et al., Z. Phys. C42 (1989) 180.

[35] Yu. L. Dokshitzer and M. Olsson, Nucl. Phys. B396 (1993) 137.

[36] W. Ochs and J. Wosiek, Z. Phys. C68 (1995) 269.

[37] DELPHI Coll., P. Abreu et al., Nucl. Phys. B444 (1995) 3.

[38] Yu. L. Dokshitzer, V. A. Khoze and S. I. Troyan, Sov. J. Nucl. Phys., 46 (1987) 712; 50 (1989) 505.

[39] S. J. Brodsky and J. F. Gunion, Phys. Rev. Lett., 37 (1976) 402.

[40] OPAL Coll., G. Alexander et al., Phys. Lett. B388 (1996) 659.

[41] V. A. Khoze, in Proceedings of the Workshop "Physics and Experiments with $e^{+} e^{-}$Linear Colliders", Saariselkä, Finland, 1991, eds. R. Orava, P. Eerola and M. Nordberg (World Scientific, Singapore) 1992, p.547.

[42] Yu. L. Dokshitzer, V. A. Khoze and S. I. Troyan, Sov. J. Nucl. Phys., 47 (1988) 881;

J. W. Gary, Phys. Rev., D49 (1994) 4503.

[43] Ya. I. Azimov, Yu. L. Dokshitzer, V. A. Khoze and S. I. Troyan, Phys. Lett., B165 (1985) 147.

[44] B. Andersson, G. Gustafson, G. Ingelman and T. Sjöstrand, Phys. Rep., 97 (1983) 31;

B. Andersson, G. Gustafson and T. Sjöstrand, Phys. Lett., B94 (1980) 211.

[45] T. Sjöstrand, Computer Physics Commun. 82 (1994) 74. 
[46] ZEUS Coll., M. Derrick et al., Phys. Lett. B348 (1995) 665; B384 (1996) 401.

[47] H1 Coll., S. Aid et al., Z. Phys. C70 (1996) 17.

[48] J.M. Butterworth, UCL/HEP 97-04, Contribution to Ringberg workshop "New Trends in HERA Physics", Tegernsee, Germany, May 1997 (hep-ex/9707001).

[49] R.K. Ellis, G. Marchesini and B.R. Webber, Nucl. Phys. 286 (1987) 643; Erratum Nucl. Phys. 294 (1987) 1180.

[50] J. Ellis, V.A. Khoze and W.J. Stirling, Z. Phys. C75 (1997) 287.

[51] V.A. Khoze and W.J. Stirling, Z. Phys. C76 (1997) 59.

[52] V.F. Andreev, V.A. Khoze and A.I. Lebedev, Sov. Phys. Lebedev Inst. Report (1989); N8, 82; V.A. Khoze, A.I. Lebedev and J.A. Vazdik, Mod. Phys. Lett., A9 (1994) 1665.

[53] T. Sjöstrand and M. van Zijl, Phys. Rev. D36 (1987) 2019.

[54] J. M. Butterworth, J. R. Forshaw, T. Sjöstrand and J. K. Storrow, J. Phys. G22 (1996) 883. 


\section{Figure captions}

Fig.1. a: Charged particle distribution $d n / d^{3} p$ as a function of particle momentum $p$. Experimental data at various $\mathrm{cms}$ energies 26, 24, 27, 25, 28] are compared with predictions using $d n / d^{3} p=2 \cdot 4 / 9 \cdot K_{h} D_{g}^{g}\left(\xi_{E}\right) /\left[4 \pi E\left(E^{2}-Q_{0}^{2}\right)\right]$ with $D_{g}^{g}$ computed in MLLA from eqs. (1, 2) $\left(Q_{0}=0.27 \mathrm{GeV}, K_{h}=0.45\right)$, which approach a common limit for $p \rightarrow 0$. The detailed shape in the dashed region is particularly sensitive to parton level approximations. b: Same distribution for charged pions [29, 27, 31, 30] in comparison with predictions using $d n / d^{3} p=2 \cdot 4 / 9 \cdot K_{h} D_{g}^{g}\left(\xi_{E}\right) /\left[4 \pi E_{h}^{3}\right]$ with $D_{g}^{g}$ computed from the Limiting Spectrum $\left(Q_{0}=0.155 \mathrm{GeV}, K_{h}=1.125\right)$.

Fig. 2. a: The same observable as in Fig. 1a, but only the data collected at cms energies from 3 up to $58 \mathrm{GeV}[26,24,32,27$ are shown. b: The same observable as in a, but only the data collected at LEP at $\mathrm{cms}$ energies $\sqrt{s}=$ 91, 133, $161 \mathrm{GeV} 25,28,31,23,33$ are shown.

Fig. 3. a: Charged particle distribution $d n / d^{3} p$ at three values of momentum $p=0.2,0.7$ and $1.2 \mathrm{GeV}$ as a function of $\mathrm{cms}$ energy $\sqrt{\mathrm{s}}$. Experimental data from $e^{+} e^{-}$annihilation experiments [26, 24, 32, 27, 25, 28, 31, 23, 33] and from ep experiment [8] in the Breit frame. In the latter case two sets of data are shown, corresponding to different ways of sampling the data (see [8]); in both sets data have been multiplied by a factor 2 to obtain a common normalization with $e^{+} e^{-}$data and a selection on the energy flow has been applied [8]. The solid line corresponds to formula (7) and the dashed one to formula (10). b: Distribution $d n / d^{3} p$ of charged kaons and of protons at fixed values of momentum as a function of $\mathrm{cms}$ energy. Experimental data from $e^{+} e^{-}$experiments 34, 31,30.

Fig. 4: Rapidity density at $y=1,\left.\frac{d n}{d y}\right|_{y=1}$, and distribution $\frac{1}{5} \frac{d n}{d^{3} p}$ at momentum $p=0.25 \mathrm{GeV}$ for charged particles in $e^{+} e^{-}$annihilation as a function of cms energy $\sqrt{s}$. Data from [19, 25, 31, 23, 33 .

Fig. 5: $m^{3} d n / d^{3} p$ distribution for charged pions, charged kaons, protons and neutral kaons in $e^{+} e^{-}$annihilation as a function of the particle momentum p. Experimental data from 29, 34, 30,31.

Fig. 6: Theoretical prediction at $\sqrt{s}=14 \mathrm{GeV}$ for the single inclusive particle density $d^{2} n / d p_{\|} d \vartheta$ as a function of the angle $\vartheta$ at four different values of $p_{\|}=0.15,0.3,0.5,0.8 \mathrm{GeV}$ (from right to left) according to MLLA and eq. (22) (with parameters $Q_{0}=0.5 \mathrm{GeV}$ and $\lambda=0.05$ ).

Fig. 7: Theoretical predictions as in Fig. 6 for $d^{2} n / d p_{\|} d p_{\perp}$ as a function of transverse momentum $p_{\perp}$ at fixed value of $p_{\|}=0.8 \mathrm{GeV}$ at four different values of $\mathrm{cms}$ energy $\sqrt{\mathrm{s}}=14$ (solid), 34 (long-dashed), 91 (short-dashed) and $172 \mathrm{GeV}$ (dotted line). 
Fig. 8: Comparison of different theoretical predictions for the $d^{2} n / d p_{\|} d p_{\perp}$ distribution as a function of transverse momentum $p_{\perp}$ at fixed value of $p_{\|}=$ $0.8 \mathrm{GeV}$ and $\sqrt{s}=34 \mathrm{GeV}$ : DLA Born term (long-dashed), DLA (shortdashed), MLLA (solid) with $Q_{0}=0.5 \mathrm{GeV}$ and $\lambda=0.05$ and Limiting Spectrum (dotted line) with the same $Q_{0}$ and $\lambda=0$ (normalization adjusted by factor $\left.K_{h}=3.5\right)$.

Fig.9: The spectrum of soft particles perpendicular to the event plane depends on the colour and relative orientation of the primary partons. 


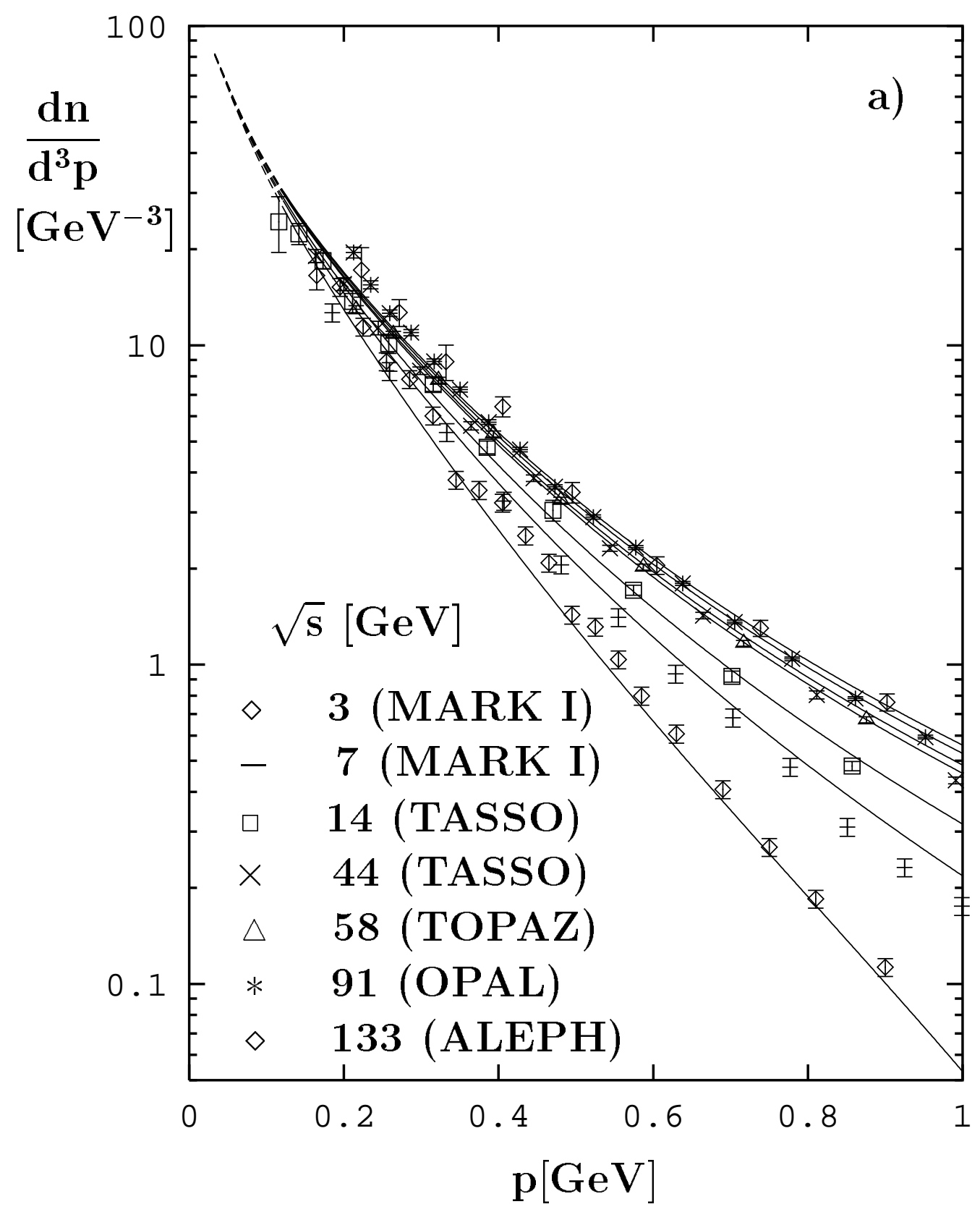

Figure 1: 


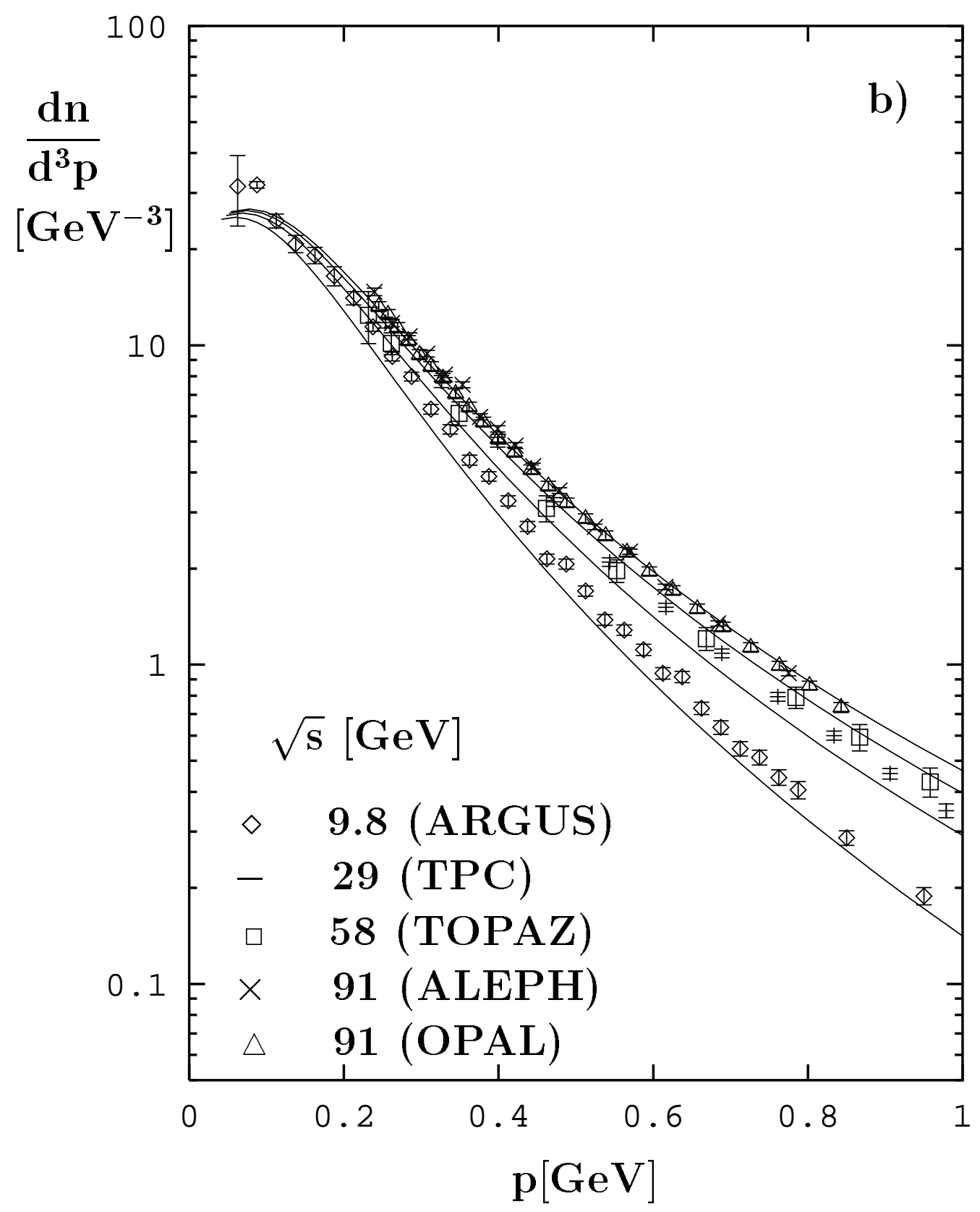

Figure 1: 


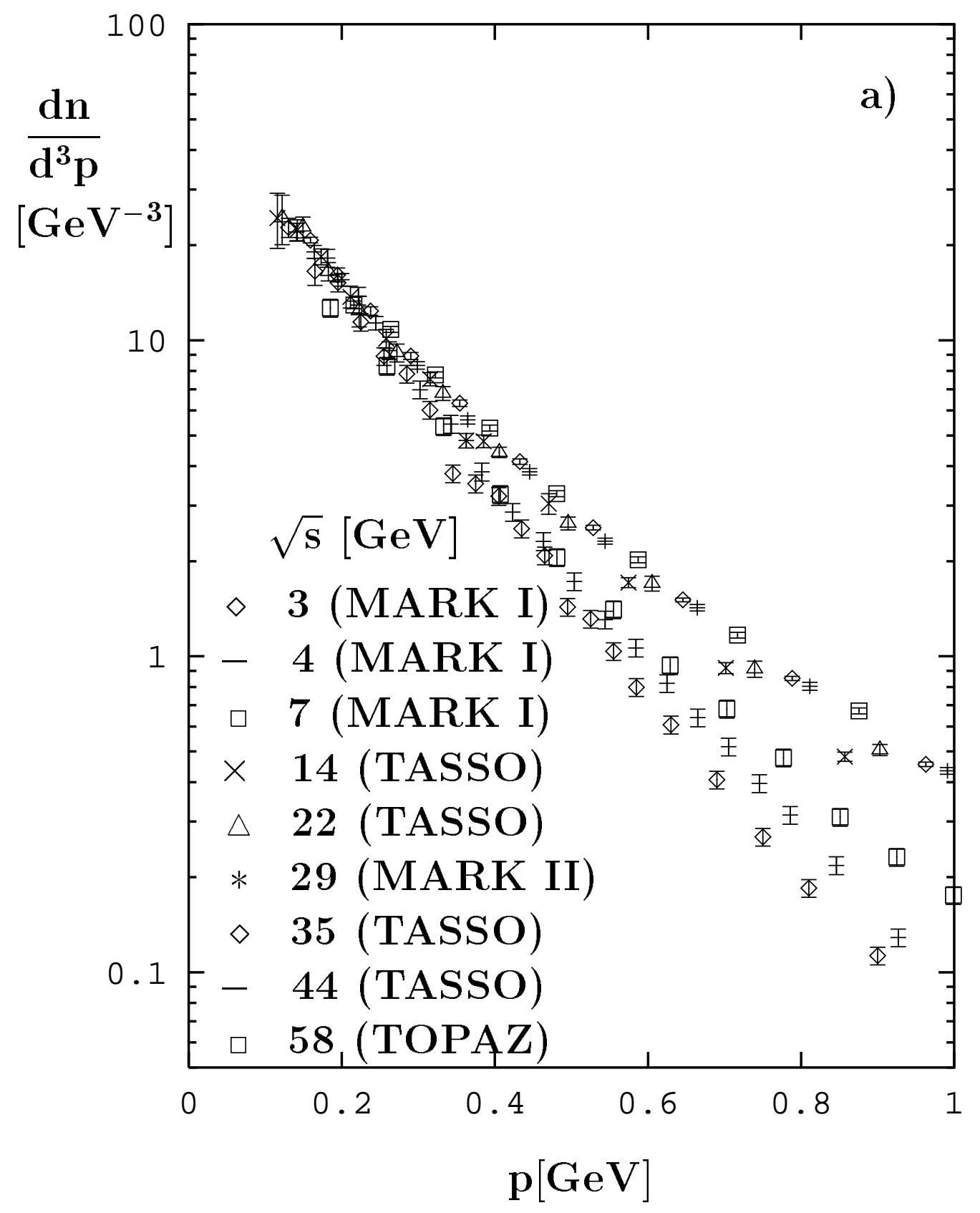

Figure 2: 


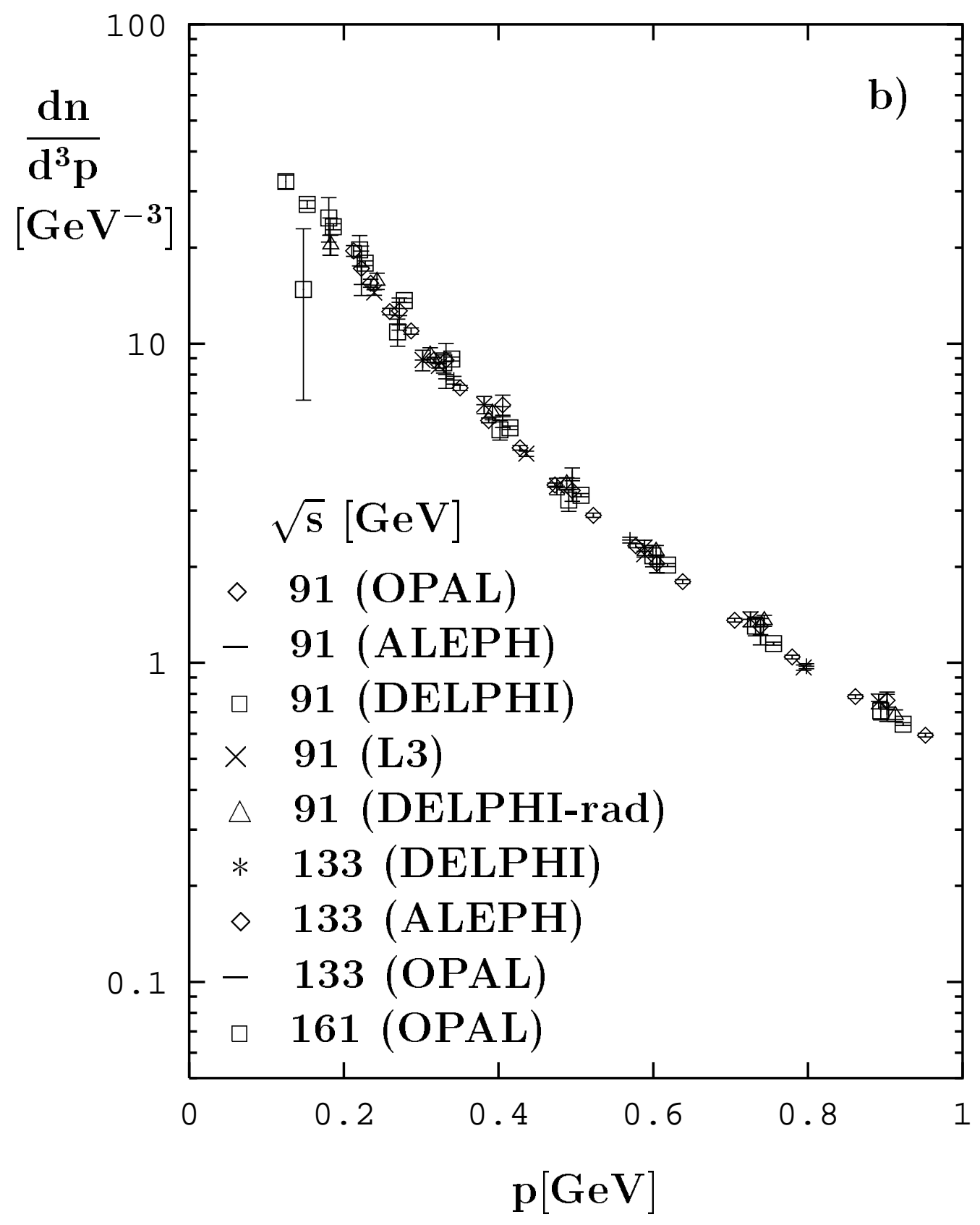

Figure 2: 


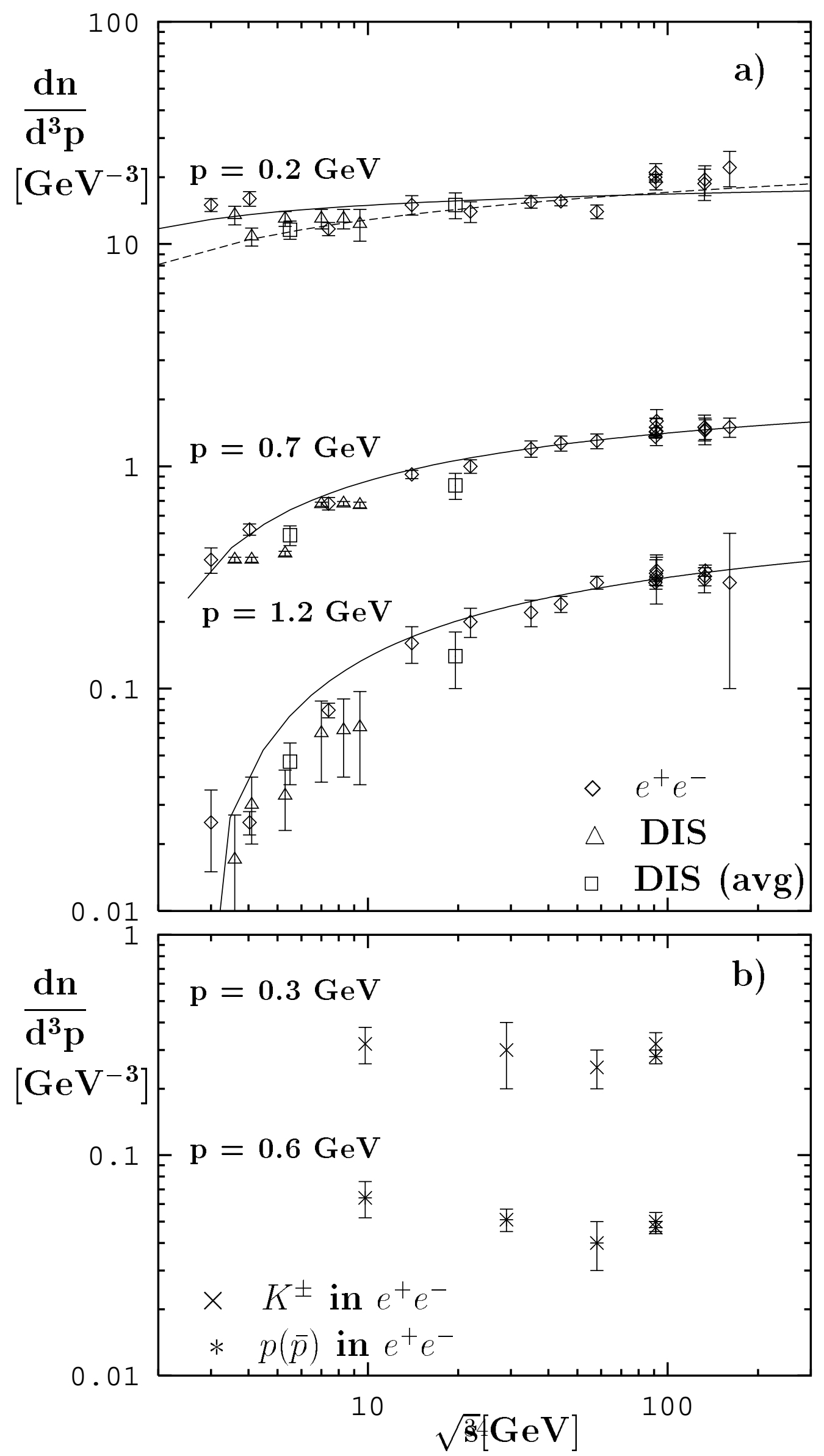

Figure 3: 


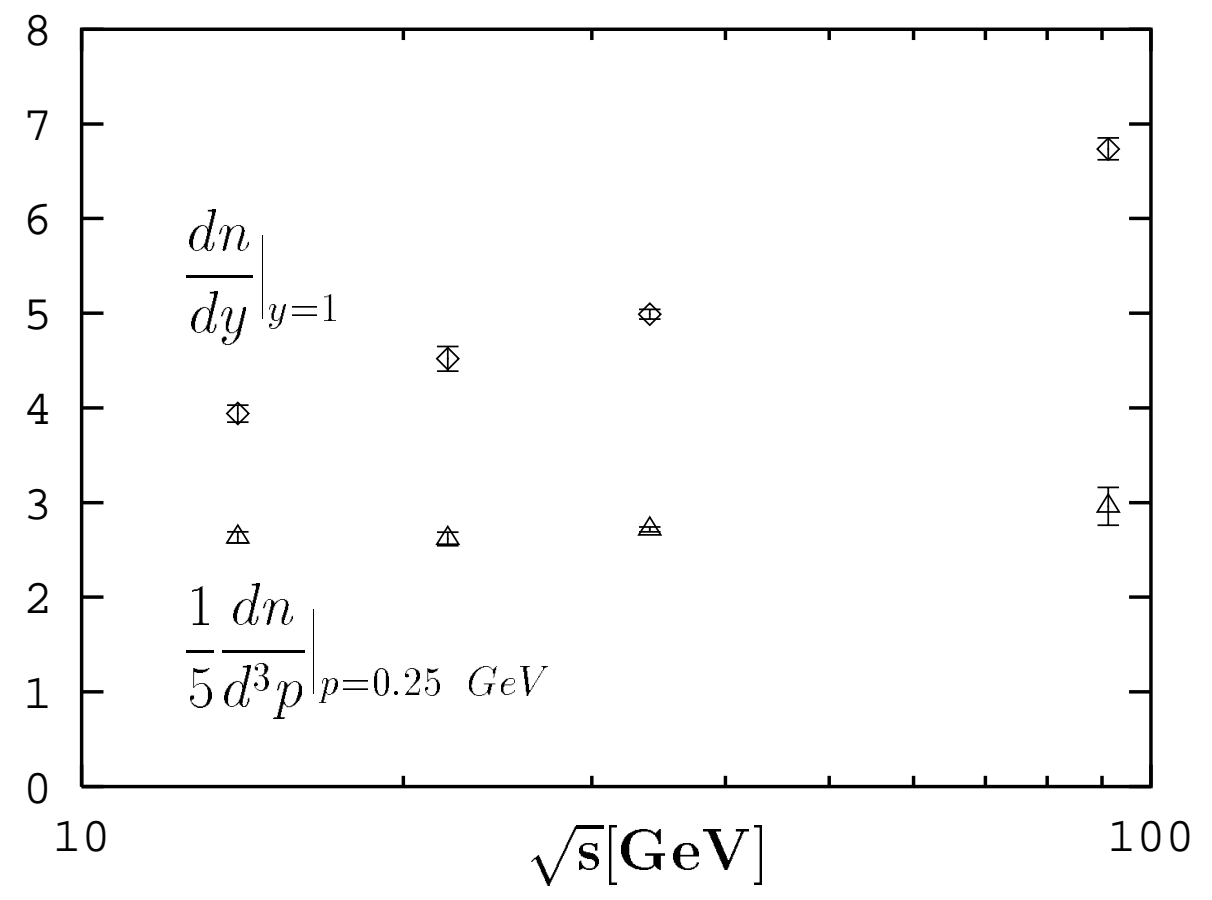

Figure 4: 


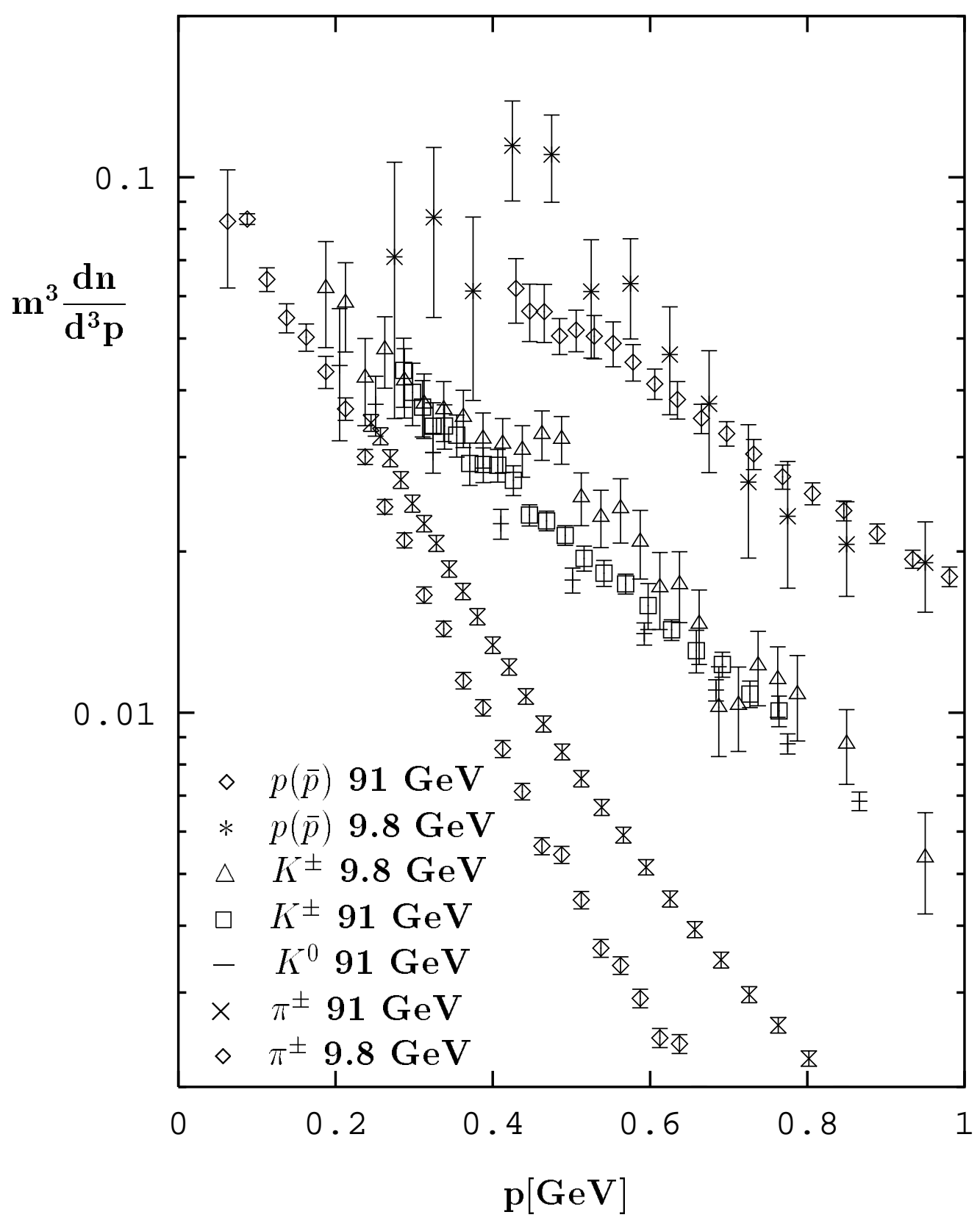

Figure 5: 


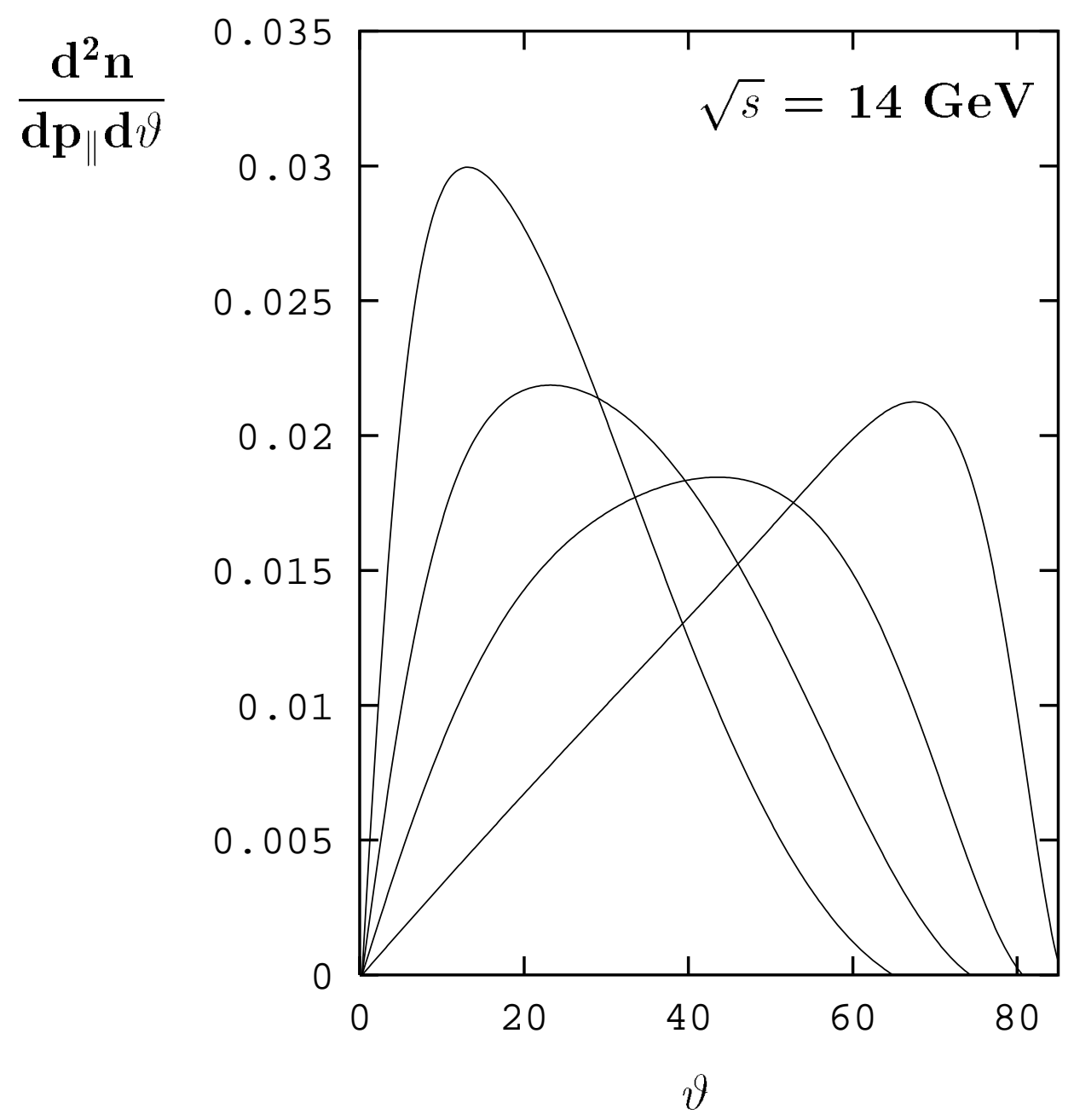

Figure 6: 


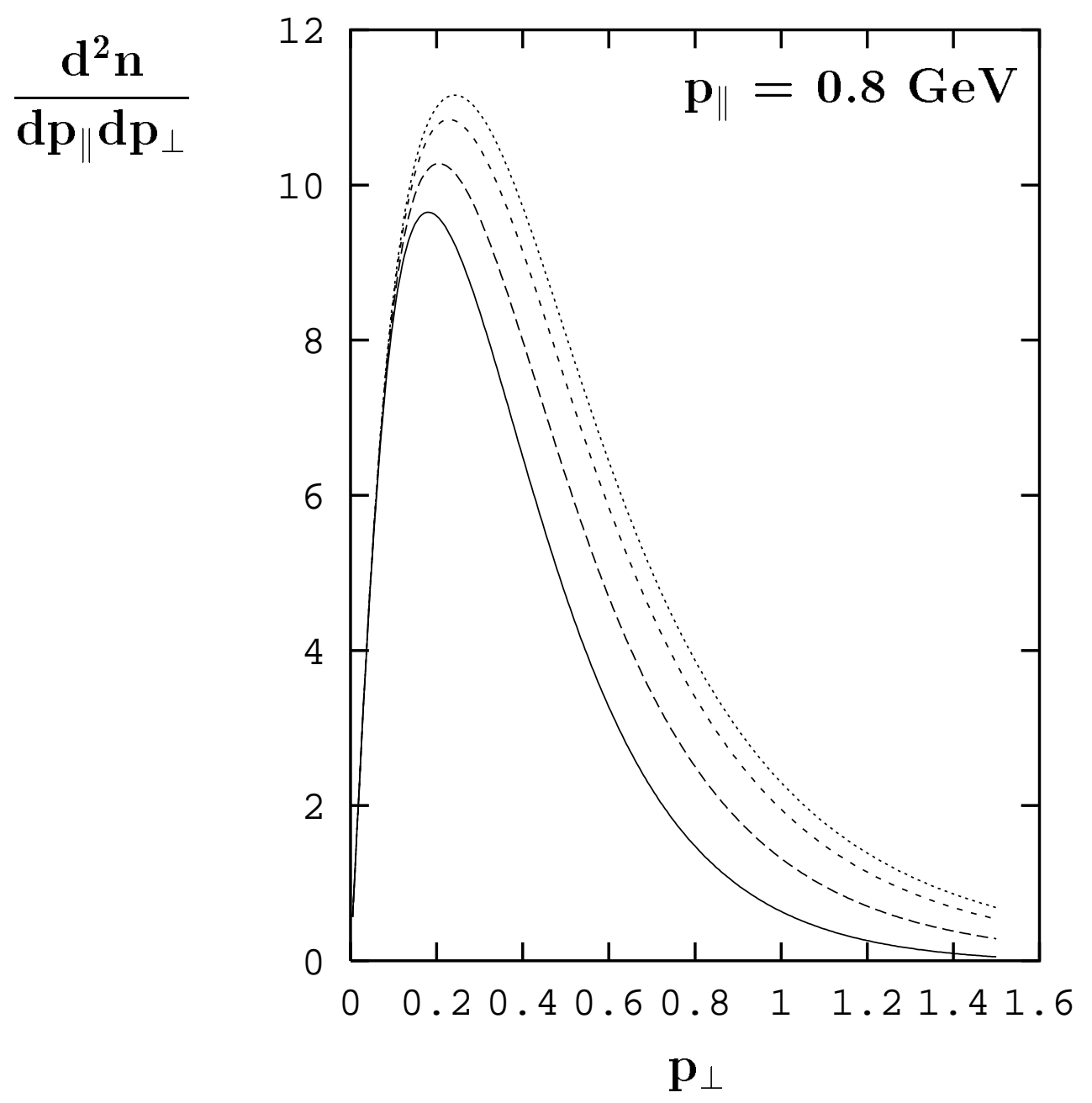

Figure 7: 


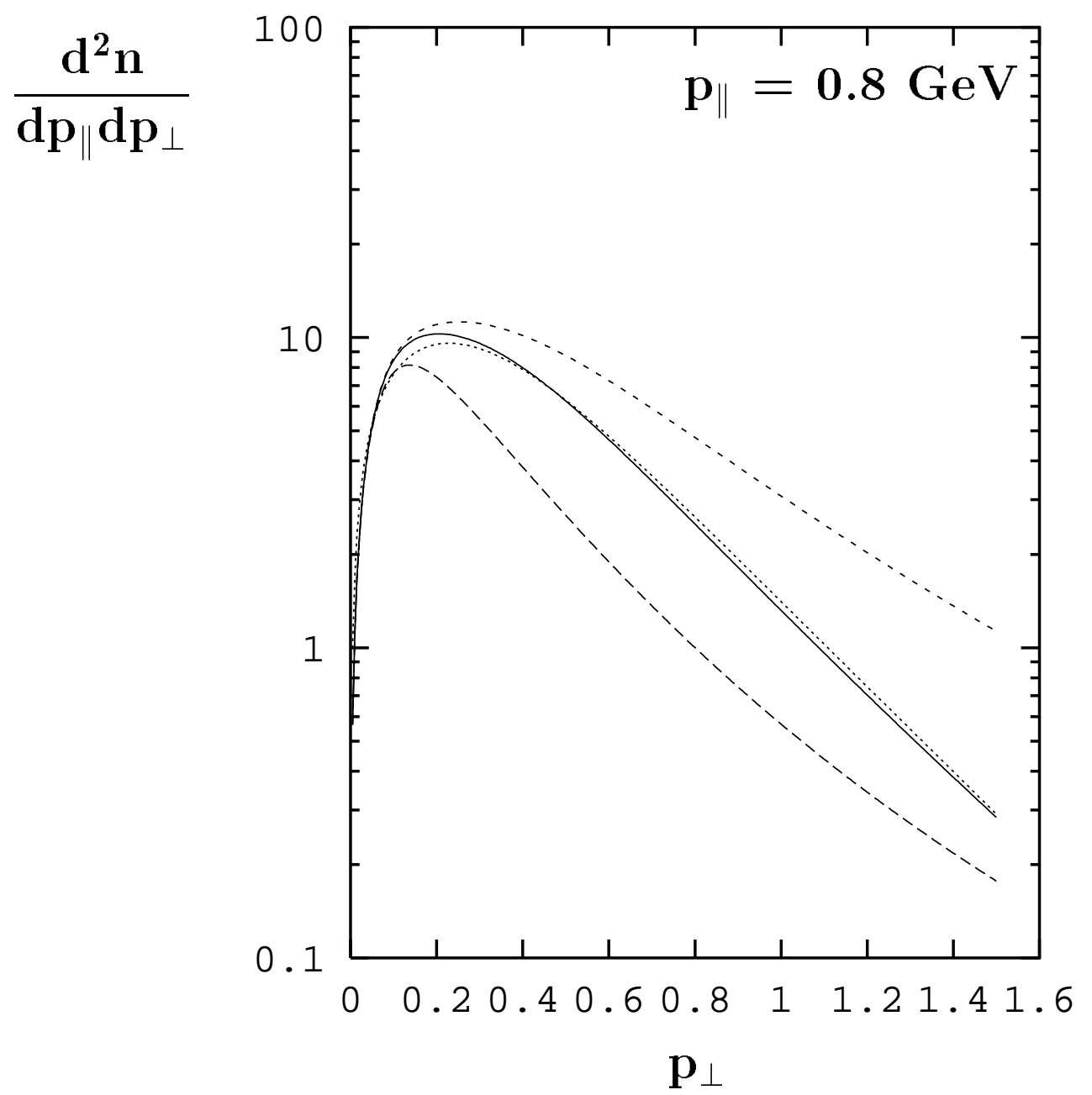

Figure 8:

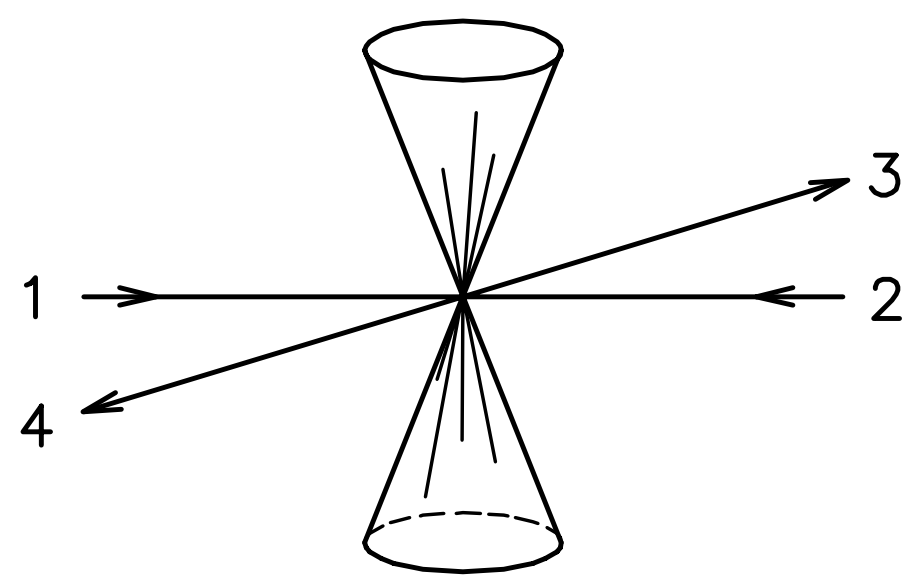

Figure 9: 